\title{
Structure of the DM Tau Outer Disk: Probing the vertical kinetic temperature gradient
}

\author{
E. Dartois ${ }^{1}$, A. Dutrey ${ }^{2}$, and S. Guilloteau ${ }^{3}$ \\ 1 Institut d'Astrophysique Spatiale, UMR 8617, Bât. 121, Campus Paris XI, 91405 Orsay, France \\ ${ }^{2}$ Laboratoire d'Astrophysique de l'Observatoire de Grenoble, BP 53, 38041 Grenoble Cedex 9, France \\ 3 Institut de Radio Astronomie Millimétrique, 300 Rue de la Piscine, 38406 Saint Martin d'Hères, France
}

Received 20 August 2002 / Accepted 6 November 2002

\begin{abstract}
We use high angular and spectral resolution $\left(\sim 1^{\prime \prime} \& 0.1 \mathrm{~km} \mathrm{~s}^{-1}\right)$ images in ${ }^{12} \mathrm{CO},{ }^{13} \mathrm{CO} J=2 \rightarrow 1, J=1 \rightarrow 0$ and $\mathrm{C}^{18} \mathrm{O} J=2 \rightarrow 1$ coming from the IRAM interferometer to probe the vertical temperature distribution in the disk of DM Tau. We investigate here a new method based on the different opacities of the $\mathrm{CO}$ isotopomers to sample the temperature disk structure at various vertical scales inside the disk. Typically, the ${ }^{12} \mathrm{CO}$ transitions are sampling at $2-4$ scale heights, while the ${ }^{13} \mathrm{CO}$ data are more likely tracing 1 scale height for the $J=2-1$, and the disk mid-plane for the $J=1-0$ line. At the disk scale at which the IRAM observations are sensitive $(R \geq 50-60 \mathrm{AU})$, the analysis reveals a vertical temperature gradient. The outer layers where $\tau\left({ }^{12} \mathrm{CO} J=2 \rightarrow 1\right) \simeq 1$ are warmer $(\sim 30 \mathrm{~K})$ than the inner layers probed by ${ }^{13} \mathrm{CO}$ and $\mathrm{C}^{18} \mathrm{O}$ data $(\sim 13-20 \mathrm{~K})$. These findings are consistent with the structure expected for flared irradiated disks around TTauri stars. We also observe that the outer radius of the disk is smaller in ${ }^{13} \mathrm{CO}$ and $\mathrm{C}^{18} \mathrm{O}$ than in ${ }^{12} \mathrm{CO}$. These differences in truncation radius are in agreement with photodissociation effects. We also note that the dynamical mass determination from $\mathrm{CO}$ is weakly affected by the temperature gradient.
\end{abstract}

Key words. stars: individual: DM Tauri - stars: circumstellar matter - stars: pre-main sequence - radio-lines: stars

\section{Introduction}

CO line observations of low-mass Pre-Main-Sequence (PMS) stars in Taurus-Auriga $(\sim 140 \mathrm{pc}$, Elias 1978) provide now strong evidence that many of them are surrounded by large $\left(R_{\text {out }} \sim 200-800\right.$ AU) Keplerian disks (GM Aur, Koerner et al. 1993; GG Tau: Dutrey et al. 1994; MWC 480: Mannings et al. 1997). Guilloteau \& Dutrey 1998 (hereafter GD98) have shown that a simple ray tracing code assuming LTE conditions applied on CO images allows one to retrieve the physical parameters of disks if the quality of the $\mathrm{CO}$ data is high enough to perform reliable $\chi^{2}$ minimisation on disk parameters. Using ${ }^{12} \mathrm{CO} J=1 \rightarrow 0$ data from the IRAM interferometer, the method was first applied to the DM Tau CO disk (GD98). One of the key parameters determined from such an analysis is the stellar mass: the initial work on DM Tau was followed by more systematic measurements for a dozen stars (Simon et al. 2000).

Another important result is the determination of the kinetic temperature law. For DM Tau, GD98 found $T_{k}(r)=$ $32(r / 100 \mathrm{AU})^{-0.63} \mathrm{~K}$. Since the ${ }^{12} \mathrm{CO} J=1 \rightarrow 0$ transition is optically thick, this measurement is relevant to the region where $\tau(\mathrm{CO})$ is around 1 . The CO column density is still very high in such objects, therefore $\tau(\mathrm{CO}) \sim 1$ is easily reached

Send offprint requests to: E. Dartois,

e-mail: emmanuel.dartois@ias.u-psud.fr and traces the "CO disk surface". This determination assumes a purely radial gradient of the temperature, and no vertical gradient. Yet, vertical gradients are expected in proto-planetary disks. Because of the frequency dependence of the dust absorption coefficient, heating by the stellar light is expected to produce an inverse green-house effect which results in a cold disk plane surrounded by a warmer disk photosphere. Detailed models have been performed by Chiang \& Goldreich (1997, hereafter CG97) and d'Alessio et al. (1999, hereafter dA99). Viscous heating resulting from accretion in the disk plane could result in the opposite effect. Imprints of such temperature gradients in the inner disk $(<10 \mathrm{AU})$ can be found in the IR SED of the disk. However, the outer disk parts are so cold that only the mm domain can probe them.

This paper provides a first attempt to measure the vertical temperature gradient in the outer parts of a proto-planetary disk. The goal is two-fold: i) to test the validity of the disk models, and ii) to assess the possible influence of such a gradient on the determination of other disk parameters (like density, inclination or dynamical stellar mass). We take advantage of the varying opacities of the lowest transitions of $\mathrm{CO}$ and its isotopes to probe different disk layers, and constrain a possible vertical temperature gradient inside the outer disk (at $r>50$ $100 \mathrm{AU})$. Such multi-transition, multi-isotope techniques were used in the past to sample temperature profiles of molecular 
cores. The extension of this "old" technique to proto-planetary disks is particularly attractive, since the known geometry of the disk allows more accurate modeling.

We describe here the method, its current limitations and give estimates of the vertical temperature gradient. We also assess the reliability of the determination of other disk parameters when the temperature gradient cannot be completely constrained. The presentation of the observations and the data reduction (in Sect. 2) is followed by a description of the method (Sect. 3). We apply it to ${ }^{12} \mathrm{CO}$ and ${ }^{13} \mathrm{CO} J=2 \rightarrow 1$ transitions in Sect. 4 . The best temperature gradient is determined by $\chi^{2}$ minimization performed on 6 different spectral lines (3 isotopes, 2 transitions). In Sect. 5, we discuss our results and compare them in Sect. 6 to temperature models existing in the literature.

\section{Observations and data reduction}

\subsection{Observations}

All observations were performed with the IRAM interferometer on Plateau de Bure. The DM Tau millimeter center is located at $\alpha(2000)=04 \mathrm{~h} 33 \mathrm{~m} 48.73 \mathrm{~s} ; \delta(2000)=18^{\circ} 10^{\prime} 10.0^{\prime \prime}$

\subsubsection{The ${ }^{12} \mathrm{CO} J=1 \rightarrow 0$ and $J=2 \rightarrow 1$ data}

The ${ }^{12} \mathrm{CO} J=1 \rightarrow 0$ data where obtained in Sep. 1995 under good weather conditions. A precise description of the observing conditions and data reduction is given in GD98. These observations provided an angular resolution of $3.5^{\prime \prime} \times 2.3^{\prime \prime}$ with a $0.17 \mathrm{~km} \mathrm{~s}^{-1}$ velocity resolution. The ${ }^{12} \mathrm{CO} J=2 \rightarrow 1$ data were obtained during the winter 1996/1997 and summer 1997. The observations were performed in snapshot mode. The observational conditions and the data reduction is described in Simon et al. (2000). The resultant angular resolution obtained is of the order of $0.7^{\prime \prime}$ with a $0.18 \mathrm{~km} \mathrm{~s}^{-1}$ velocity resolution. In one transit, 4 T Tauri disks where observed. The total integration time on the DM Tau disk is about 4 hours.

\subsubsection{The ${ }^{13} \mathrm{CO} J=1 \rightarrow 0$ data}

The ${ }^{13} \mathrm{CO} J=1 \rightarrow 0$ line was observed in $\mathrm{C} 2, \mathrm{~B} 2$ and $\mathrm{A}$ configurations between Feb. 1999 and March 1999. This provides baselines up to $400 \mathrm{~m}$, and an angular resolution of $1.73^{\prime \prime} \times 1.63^{\prime \prime}$ at PA $7^{\circ}$ at $2.7 \mathrm{~mm}$, after tapering to adjust to the same resolution as the $J=2 \rightarrow 1$ line. We observed simultaneously at $110 \mathrm{GHz}$ to cover the ${ }^{13} \mathrm{CO} J=1 \rightarrow 0$ and $\mathrm{C}^{18} \mathrm{O} J=1 \rightarrow 0$ lines, and at $220 \mathrm{GHz}$ to cover the $\mathrm{C}^{18} \mathrm{O} J=2 \rightarrow 1$ line. At $1.3 \mathrm{~mm}$, the tuning was double-sideband (DSB) while at $2.7 \mathrm{~mm}$, the tuning offered $6 \mathrm{~dB}$ rejection. The rms phase were up to $40^{\circ}$ at $2.7 \mathrm{~mm}$ on the longest baseline, offering a seeing better than $0.7^{\prime \prime}$. The backend correlator of $10 \mathrm{MHz}$ bandwidth provided a velocity resolution of $0.11 \mathrm{~km} \mathrm{~s}^{-1}$

\subsubsection{The $\mathrm{C}^{18} \mathrm{O} J=2 \rightarrow 1$ and $J=1 \rightarrow 0$ data}

The $\mathrm{C}^{18} \mathrm{O} J=2 \rightarrow 1$ line was observed in C2, B2 and A configurations between Feb. 1999 and March 1999, simultaneously with the ${ }^{13} \mathrm{CO} J=1 \rightarrow 0$ and $\mathrm{C}^{18} \mathrm{O} J=1 \rightarrow 0$ lines. The data was heavily tapered to produce an image with an angular resolution of $1.71^{\prime \prime} \times 1.54^{\prime \prime}$ at PA $9^{\circ}$. We also applied Hanning smoothing in velocity to obtain $0.23 \mathrm{~km} \mathrm{~s}^{-1}$ resolution, with $0.11 \mathrm{~km} \mathrm{~s}^{-1}$ channel spacing. The $\mathrm{C}^{18} \mathrm{O} J=1 \rightarrow 0$ line was also detected during these observations, but does not bring any new significant constraint due to the low signal to noise of this detection.

\subsubsection{The ${ }^{13} \mathrm{CO} J=2 \rightarrow 1$ data}

The ${ }^{13} \mathrm{CO} J=2 \rightarrow 1$ line was observed in D configuration on Nov. 28, 1999 and in C2 configurations on Jan. 29 and Feb. 11, 2001. This provides baselines up to $180 \mathrm{~m}$, and an angular resolution of $1.73^{\prime \prime} \times 1.59^{\prime \prime}$ at PA $27^{\circ}$, nearly identical to that of the ${ }^{13} \mathrm{CO} J=1 \rightarrow 0$ data. We observed simultaneously at $88 \mathrm{GHz}(\mathrm{HCN} J=1 \rightarrow 0)$ and $220.4 \mathrm{GHz}\left({ }^{13} \mathrm{CO} J=2 \rightarrow 1\right)$. At $1.3 \mathrm{~mm}$, the tuning was double-side-band (DSB) while at $3.4 \mathrm{~mm}$, the tuning was purely single-side band (LSB). The backend was a correlator with one band of $10 \mathrm{MHz}$ (spectral resolution $0.13 \mathrm{~km} \mathrm{~s}^{-1}$ ) centered on the $\mathrm{HCN} J=1 \rightarrow 0$ line, and one band of $20 \mathrm{MHz}$ (spectral resolution $0.13 \mathrm{~km} \mathrm{~s}^{-1}$ ) centered on the ${ }^{12} \mathrm{CO} J=2 \rightarrow 1$ line respectively. The remaining bands were used to provide continuum observations. The phase and flux calibrators were $0415+379,0430+052$ and $0528+134$, and the absolute flux scale was set using MWC 349 as a reference. The rms phase noise was $7^{\circ}$ to $20^{\circ}$ and $15^{\circ}$ to $50^{\circ}$ at $3.4 \mathrm{~mm}$ and $1.3 \mathrm{~mm}$, respectively, resulting in an effective seeing about $0.7^{\prime \prime}$.

\subsection{Data reduction}

We used the GILDAS software package to reduce the data. Images were produced using various tapers, to match all images to similar angular resolution. However, in the model fitting, the original, untapered $u v$ distributions were used. We did not subtract the continuum emission from the line maps. The ${ }^{13} \mathrm{CO} J=1 \rightarrow 0,{ }^{13} \mathrm{CO} J=2 \rightarrow 1$ and $\mathrm{C}^{18} \mathrm{O} J=2 \rightarrow 1$ maps are presented in Fig. 1.

The total line flux is an important parameter to constrain the temperature of the disk (see Sect. 3). Therefore having a coherent absolute calibration scale for every line observed is fundamental. Although our original calibration is expected to be accurate to the $15 \%$ level or better, in order to optimize the flux calibration, we proceed as follows. We calibrate the flux density by the standard method using MWC 349 (IRAM flux report 13) and the quasars $0415+379,0528+134$ or $3 \mathrm{C} 273$ as secondary references. We then measured the total flux density of the dust disk at the various observing wavelengths: $3.5,3.4$, $2.7,2.6,1.35,1.3 \mathrm{~mm}$. We find that the best spectral index for the dust disk is $\alpha=2.6 \pm 0.1$. Then assuming $\alpha=2.6$ and using the ${ }^{12} \mathrm{CO} J=2 \rightarrow 1$ frequency for the absolute flux reference $(101 \pm 2 \mathrm{mJy}$ ), we fix the continuum flux (hence the line flux) to the fitted values. No correction higher than $15 \%$ was found in this process. This flux density scale is accurately transferred to the line observations due to the high precision of the bandpass calibrations $(\simeq 1 \%)$. By this method, we obtain a reliable 

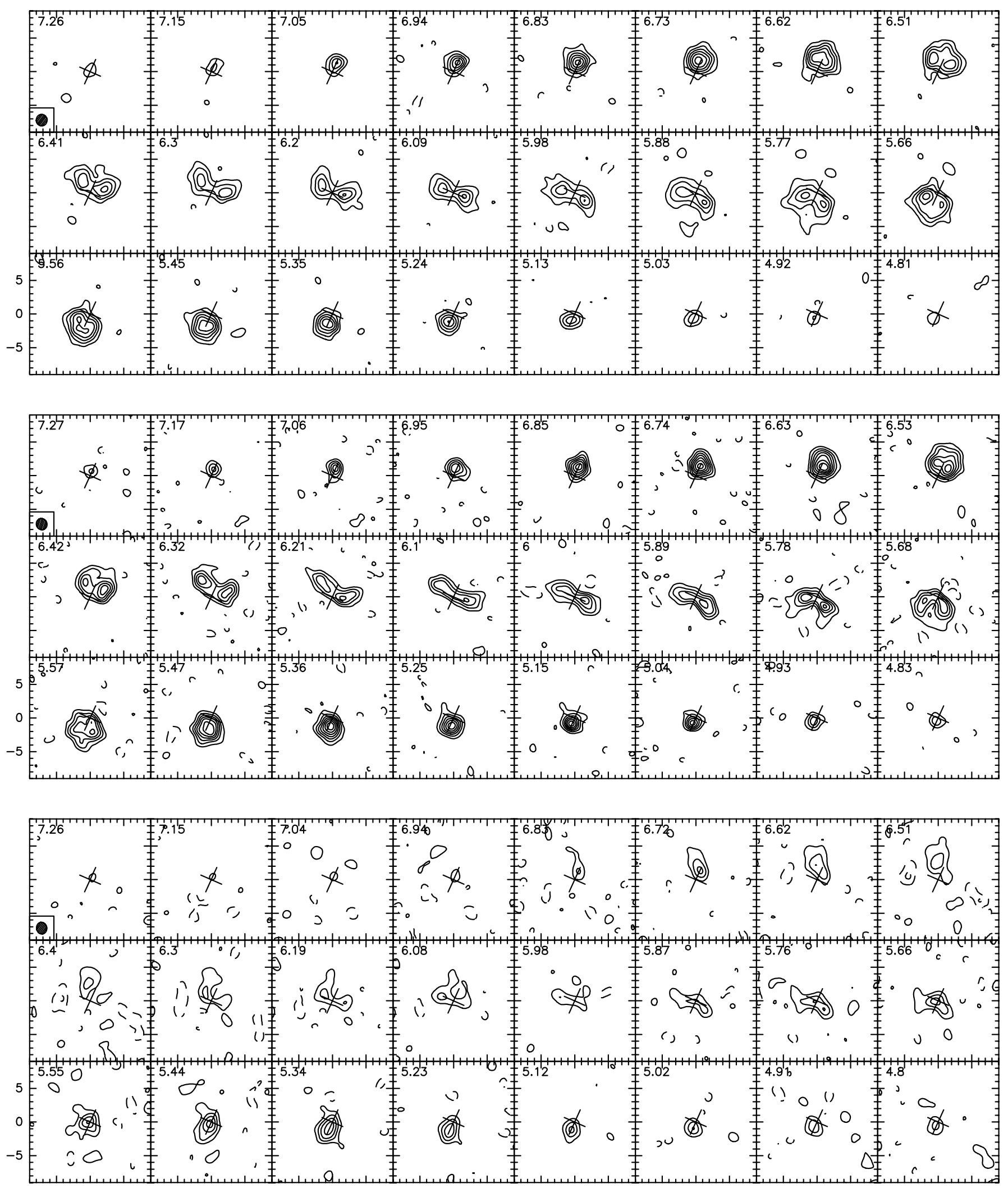

Fig. 1. Channel maps of the ${ }^{13} \mathrm{CO} J=1 \rightarrow 0$ (upper panels), ${ }^{13} \mathrm{CO} J=2 \rightarrow 1$ (middle panels) and $\mathrm{C}^{18} \mathrm{O} J=2 \rightarrow 1$ (bottom panels) in DM Tau. For ${ }^{13} \mathrm{CO}$, the contours are spaced by $1 \mathrm{~K}$ (approximately $2 \sigma$ ), while for $\mathrm{C}^{18} \mathrm{O}$ the spacing is $0.5 \mathrm{~K}$ (about $1.5 \sigma$ ). All spatial resolutions are similar $\left(1.7^{\prime \prime} \times 1.6^{\prime \prime}\right.$ at PA $\left.10^{\circ}\right)$. The $\mathrm{C}^{18} \mathrm{O} J=2 \rightarrow 1$ data has been Hanning smoothed in velocity to $0.23 \mathrm{~km} \mathrm{~s}^{-1}$. The cross indicates the position and orientation (major and minor axis) of the disk. 
relative calibration from one frequency to another, thereby allowing direct comparison of the temperatures and molecular abundances determined by the various observations. Note also that the flux scale obtained with this method agrees within one $\sigma$ with the bolometer flux determination at $1 \mathrm{~mm}$ from Beckwith et al. (1990) $(109 \pm 13 \mathrm{mJy})$.

\section{Probing the gas temperature gradient: Method}

$\mathrm{CO}$ presents the advantage to possess 4 isotopomers: ${ }^{12} \mathrm{CO}$, ${ }^{13} \mathrm{CO}, \mathrm{C}^{18} \mathrm{O}$ and $\mathrm{C}^{17} \mathrm{O}$, whose relative abundances in disks span a large range. The various transitions of each species, each one having different line strengths, provide an access to different depth levels inside the disk. The analysis of the $J=1 \rightarrow 0$ and $J=2 \rightarrow 1$ rotational lines of $\mathrm{CO}$ are also simplified by the fact that they have low critical densities and are therefore thermalized at the densities encountered in protoplanetary disks (this can be seen in Fig. 9 where we plot $n_{\mathrm{c}}$ for the $J=2 \rightarrow 1$ transition of $\mathrm{CO}$ ). Based on these ideas, using the LTE excitation radiative transfer model by Dutrey et al. (1994), we can determine the surface at which $\tau=1$ is reached along the line-ofsight, for the various $\mathrm{CO}$ isotopomers and transitions $J=2 \rightarrow 1$ and $J=1 \rightarrow 0$. More details about this model can be found in the appendix.

In a first step, following GD98, we assumes that there is no dependency of $T_{k}$ with $z$, the vertical scale of the disk. In this model, the kinetic temperature only varies as a power law with radius $r$ like $T_{k}(r)=T_{\mathrm{o}} \times\left(r / r_{\mathrm{o}}\right)^{-q}$. For convenience, we call Type 0 this isothermal model. The input parameters of the model are given by the $\chi^{2}$ minimization on ${ }^{12} \mathrm{CO} J=1 \rightarrow 0$ channel maps presented by GD98 (their Table 1).

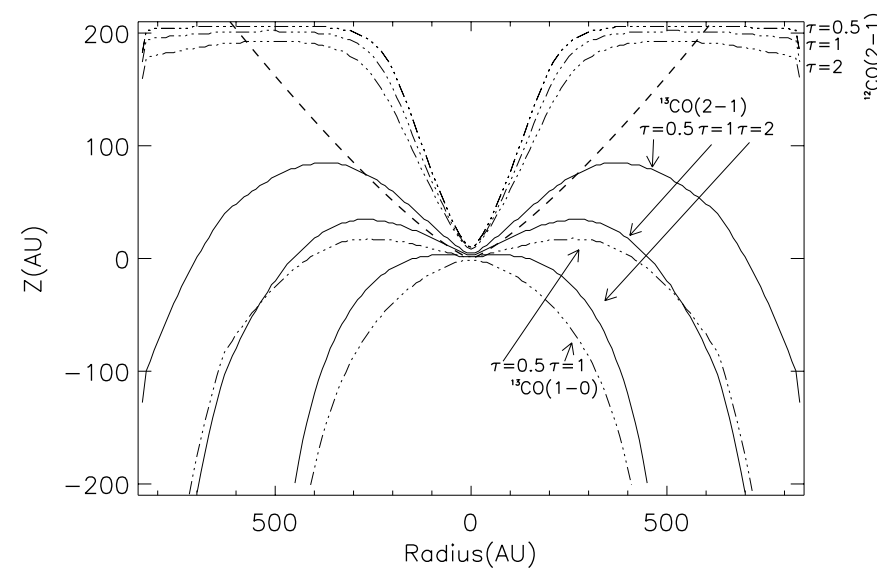

Fig. 2. Iso-opacity surfaces for various $\mathrm{CO}$ and ${ }^{13} \mathrm{CO}$ transitions calculated using the model of Dutrey et al. (1994) (type 0) in the case of the DM Tau disk as seen pole-on (observer on top). The $X$-axis is a cut in a direction perpendicular to the disk, the $Y$-axis representing the altitude in the "atmosphere" of the disk. The $\tau=1$ surfaces shows from where the contribution to the observed emission comes from. The surfaces at $\tau=0.5$ and 2 delineates the narrowness of this emitting region. ${ }^{12} \mathrm{CO} J=2 \rightarrow 1$ and $J=1 \rightarrow 0$ transitions probe the surface of the disk, ${ }^{13} \mathrm{CO} J=2 \rightarrow 1$ the mid-plane whereas ${ }^{13} \mathrm{CO} J=1 \rightarrow 0$ becomes optically thin (as it never reaches $\tau=2$ ). For comparison the dotted line represent the local density scale height $H$.

\subsection{Pole-on disk}

Let us assume first that the disk is seen pole-on (the inclination angle $i=0$ ). Figure 2 displays the vertical scale $z$ at which $\tau=$ $0.5,1$ and 2 are reached for the observed transitions $\left({ }^{12} \mathrm{CO} J=\right.$ $2 \rightarrow 1,{ }^{13} \mathrm{CO} J=2 \rightarrow 1$ and ${ }^{13} \mathrm{CO} J=1 \rightarrow 0$ ) as a function of $r$. The physical parameters used to derive these curves are appropriate for a disk like that of DM Tau (including the CO abundance). The surfaces at $\tau=0.5$ and 2 , are given in order to indicate the narrowness of the emitting region. By comparison to typical planetary atmospheres, this is equivalent to estimate how wide is the atmospheric weight function in the disk.

Figure 2 clearly shows that the ${ }^{12} \mathrm{CO} J=2 \rightarrow 1$ and $J=$ $1 \rightarrow 0$ emissions originate from a narrow region in the very upper part of the disk. It is then not surprising that the best models of ${ }^{12} \mathrm{CO} J=1 \rightarrow 0$ and $J=2 \rightarrow 1$ maps give exactly the same disk parameters (see Simon et al. 2000): both transitions of ${ }^{12} \mathrm{CO}$ are sampling the same vertical scale. In the central regions $(r<200 \mathrm{AU})$, the ${ }^{12} \mathrm{CO}$ surface is located well above the hydrodynamic scale height $H$ (thick point line), at which $n(r, H)=\frac{1}{\mathrm{e}} n(r, z=0)$ (see also appendix).

Contrary to ${ }^{12} \mathrm{CO}$, the main contribution to the ${ }^{13} \mathrm{CO} J=$ $2 \rightarrow 1$ emission arises near the disk plane. The $J=1 \rightarrow 0$ transition starts to be almost optically thin as the surface at $\tau=1$ crosses the mid-plane, and the density then falls exponentially. Both transitions sample a much thicker part of the disk than the ${ }^{12} \mathrm{CO}$ lines.

\subsection{Effect of the inclination angle $i$}

For $i \neq 0$, as it is usually the case, the existence of gradients in density allows us to sample various vertical scales $z$ in the disk depending which velocity is observed. The density gradients encountered in the disk imply that at a given velocity and projected distance $d$ to the star, one does not penetrate through the disk atmosphere symmetrically. This implies that, even in the case of an isothermal vertical atmosphere, the emission from the surface at $\tau=1$ in the two symmetric lines-of-sight associated to $d$ does not come from the same height $z$ in the disk.

This property is illustrated in Fig. 3, where we performed the same calculations as above with $i=33^{\circ}$, the inclination measured in DM Tau by GD98. The surfaces at $\tau=1$ are shown for the main transitions of ${ }^{12} \mathrm{CO}$ and ${ }^{13} \mathrm{CO}$ in various cuts through the more representative velocity channels. We chose three main channels: (a) the systemic velocity of the disk; (b) an intermediate channel; (c) an extreme channel probing the inner part of the disk. Therefore a wealth of information on the vertical temperature distribution can come from the observation of a single transition. Indeed, the comparison between a model and the data, based on a $\chi^{2}$ minimization (as done by GD98) use all the spectral and spatial information originating in various parts of the disk to constrain the temperature model. GD98 measure that the temperature follows $T_{k}(r)=32 \times(r / 100 \mathrm{AU})^{-0.63}$. The curves presented in Fig. 3 show that this measurement corresponds to scales between 50 and $200 \mathrm{AU}$ above the disk plane. 

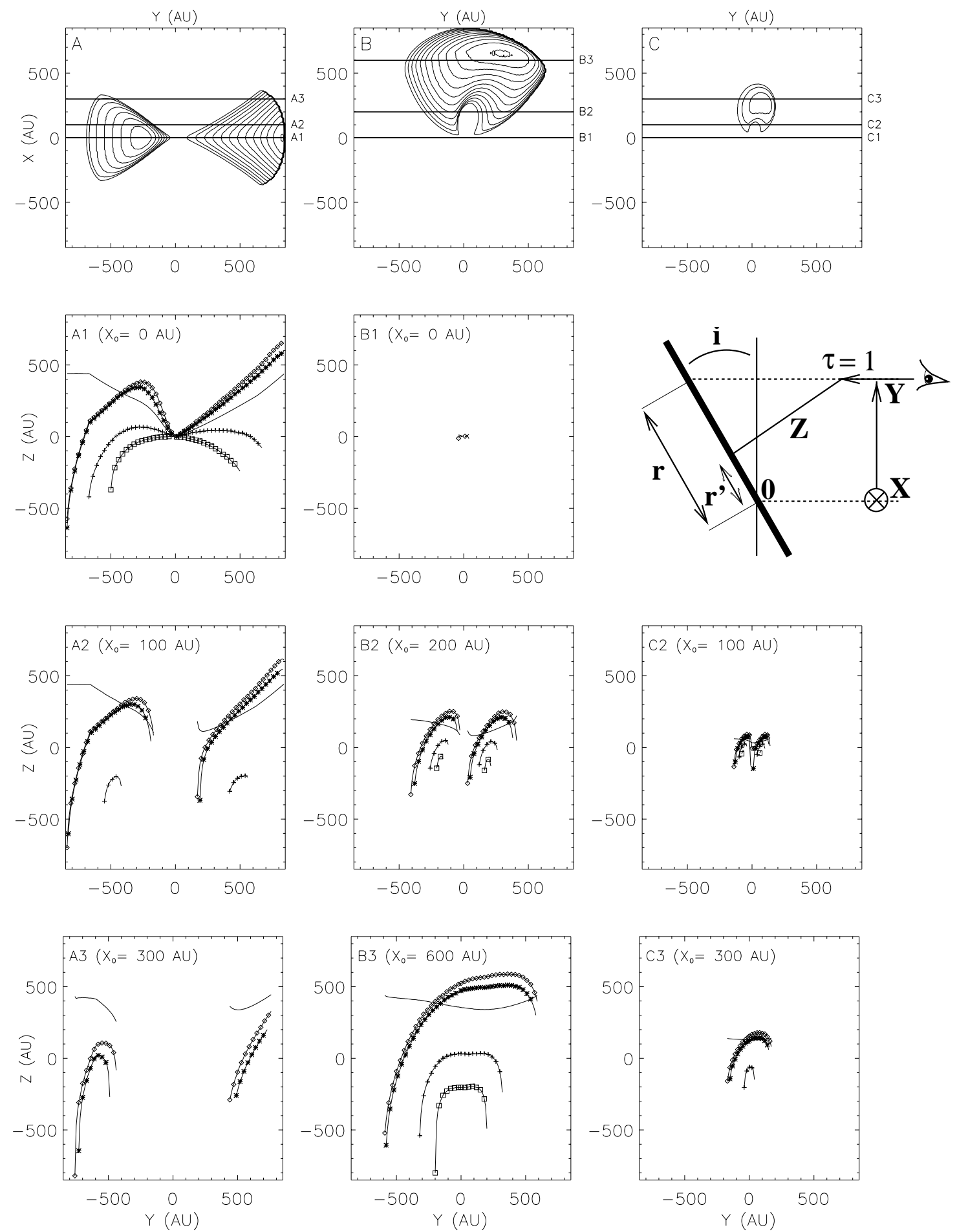

Fig. 3. Representation of the calculated iso-opacity surfaces in three characteristic velocity channels of the ${ }^{12} \mathrm{CO}$ and ${ }^{13} \mathrm{CO}$ transitions for the DM Tau disk, inclined at $33^{\circ}$ on the line of sight (type 0). From left to right are (A) the disk systemic velocity (B) $0.44 \mathrm{~km} \mathrm{~s}{ }^{-1}$ and (C) $0.77 \mathrm{~km} \mathrm{~s}^{-1}$ from this channel. The upper contour plots are the ${ }^{12} \mathrm{CO} J=2 \rightarrow 1$ height above plane at which $\tau=1$. The contours start at $25 \mathrm{AU}$ and increase by the same amount. The figures below shows cuts along the lines labeled A1 to A3, B1 to B3 and C2, C3 shown in the upper panels, and represent the various isotopes and transitions. From the upper to lower curve are the ${ }^{12} \mathrm{CO} J=2 \rightarrow 1$ (diamonds), $J=1 \rightarrow 0$ (stars) then ${ }^{13} \mathrm{CO} J=2 \rightarrow 1$ (crosses), and $J=1 \rightarrow 0$ (squares) transitions, in the cut passing through the center in the systemic velocity channel. The full line display the local scale height. The insert on the right explains the notation used in the projections shown here, where the thicker line represents the disk midplane. 


\subsection{The $\mathrm{CO}$ isotopes}

While ${ }^{12} \mathrm{CO}$ samples at $\sim 2-4$ scale heights from the disk midplane, with the $\mathrm{CO}$ isotopes, in particular through the ${ }^{13} \mathrm{CO} J=$ $1 \rightarrow 0$ line, one can sample the disk properties much closer to the disk plane (see Figs. 2 and 3). However, part of the emission becomes optically thin, so that not only the temperature, but also the surface density (and chemical abundance) governs the brightness temperature. On one hand, this gives a chance to constrain the surface density, on another hand, the analysis becomes more complex since parameters like $(p, q)$ are coupled, as are $X(\mathrm{~mol}), \Sigma_{0}$ and $T_{0}$.

In the case of the ${ }^{13} \mathrm{CO} J=1 \rightarrow 0$ transition, this coupling takes a particularly simple form, because, to first order, the line is optically thin throughout most of the disk. For a face on disk, the brightness temperature is

$$
\begin{aligned}
T b(r) & =\left(1-\mathrm{e}^{-\tau(r)}\right) T_{\mathrm{ex}}(r) \simeq \tau(r) T_{k}(r) \\
\tau(r) & \propto\left(1-\mathrm{e}^{-h v_{10} / k T_{k}(r)}\right) \frac{X(\mathrm{~mol}) \Sigma(r)}{Z \Delta V} \\
& \propto \frac{X(\mathrm{~mol}) \Sigma(r)}{Z \Delta V} \frac{h v_{10}}{k T_{k}(r)}
\end{aligned}
$$

with the proportionality factor depending on the molecular parameters only. $\Delta V$ is the local line width, and $Z$ is the partition function, given by:

$Z \approx \sum_{J=0}^{\infty}(2 J+1) \mathrm{e}^{-J(J+1) h v_{10} / k T_{k}}$.

In the high temperature limit, the partition function can be approximated by:

$Z \approx \frac{2 k T_{k}(r)}{h v_{10}}$

since CO is thermalized. Simplifying Eqs. (1)-(5) gives

$$
\begin{aligned}
T b(r) & \propto X(\mathrm{~mol}) \Sigma(r) /\left(T_{k}(r) \Delta V\right) \\
& \left.\propto X(\mathrm{~mol}) \frac{\Sigma_{0}}{T_{0}^{3 / 2}} r^{-p+3 q / 2} \quad \text { (thermal: } \Delta V \propto \sqrt{T_{k}}\right) \\
& \propto X(\mathrm{~mol}) \frac{\Sigma_{0}}{T_{0}} r^{-p+q} \quad \text { (constant linewidth) } .
\end{aligned}
$$

Thus, for an optically thin $J=1 \rightarrow 0$ line, in the approximation of constant linewidth and constant abundance, the brightness temperature would only depend on $\Sigma / T$ and hence would constrain $p-q$.

This is not the case for the $J=2 \rightarrow 1$ line because 1) of the exponential dependence on the temperature $T_{k}(r)$ of the population of the lower level, but also because 2) this transition is optically thick out to about 400 AU. The ${ }^{13} \mathrm{CO} J=1 \rightarrow 0$ data only constrain $p-q$ and $\Sigma / T$, while the ${ }^{13} \mathrm{CO} J=2 \rightarrow 1$ data offer a more complex dependence on the parameters.

These behaviors are shown in Fig. 4 where are displayed the exact and high temperature limit calculations, for the three first transitions of $\mathrm{CO}$ as a function of the gas kinetic temperature. Note that in the $10-20 \mathrm{~K}$ range the $\mathrm{CO} J=2 \rightarrow 1$ line brightness temperature only varies by $10 \%$, as does the $J=3 \rightarrow 2$ one in the $15-40 \mathrm{~K}$ range.

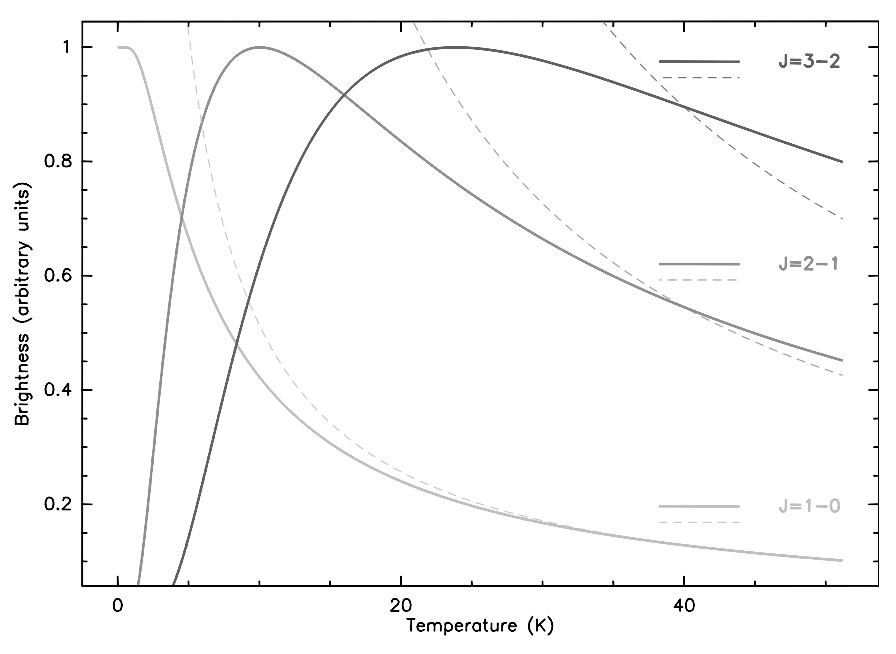

Fig. 4. Dependence of the brightness temperature (arbitrary units) versus kinetic temperature for the $J=1-0,2-1$ and 3-2 CO transitions in the constant linewidth and optically thin cases. Thick lines use the exact calculation. Dashed lines correspond to the high temperature approximation limit, where the brightness follows a $1 / T$ dependence.

\section{Vertical temperature gradient}

\subsection{Disk parametrization}

The parametric disk model used by GD98 does not take into account a possible vertical variation of the temperature. The dA99 model shows that the temperature distribution of disks as a function of radius is quite complex (see their Fig. 3). A parametric approach to such a complex variation is not simple, and would require a significant number of new free parameters. Rather than following such an approach, we shall first show that information on a vertical temperature gradient can be recovered from parametric disk models which assume only radial gradients. This is feasible because vertical temperature gradients imply different radial temperature distributions as function of scale height in the disk (see for example Fig. 5). We would then expect that the analysis of the various $\mathrm{CO}$ isotopes and transitions will result in different values for $T_{\mathrm{o}}$ and $q$ (and perhaps some other disk parameters).

To illustrate this property, and to find out which are the relevant observable parameters for a possible temperature variation, we generated two disk models with different vertical temperature gradients.

\section{Type I}

The first one (type I) is a very simple modification of the type 0 temperature distribution $T_{k}(r)=T_{\mathrm{o}}\left(\frac{r}{100 \mathrm{AU}}\right)^{-q}$ by adding a single parameter $\gamma$ :

$T_{k}(r, z)=T_{k}(r, z=0) \times \mathrm{e}^{\ln (\gamma) \cdot \frac{z}{H}}$

$H$, the hydrostatic scale height, is calculated with the temperature at $z=0$ where $T_{k}(r, z=0)=T_{\mathrm{o}}\left(\frac{r}{100 \mathrm{AU}}\right)^{-q}$ and $\gamma$ is the index of the vertical law. Note that $\ln (\gamma)$ can be either positive or negative, $\gamma=1$ means no vertical gradient (type 0 ) and $\gamma=2$ implies that at $z=H$ the temperature has reached twice its value in the midplane $(z=0)$. 


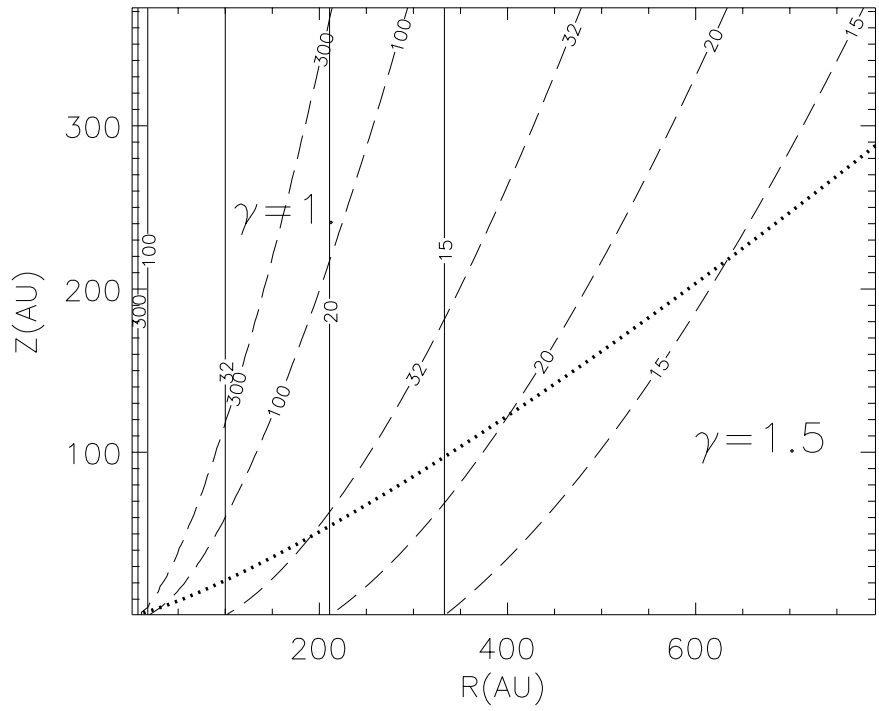

Fig. 5. Representation of the isothermal curves in the case of a purely radial dependence of the temperature distribution $(\gamma=1$ : type 0 , thin line) and when a vertical temperature gradient is included $(\gamma=1.5$ : type I, dashed line). See text for the adopted gradient parameterization. The dotted line shows the local hydrostatic scale height.

Although it does not necessarily bear any physical justification, this simple model can simulate either positive gradients (hotter disk atmosphere, like in CG97, see also Sect. 6) or negative gradients (hotter disk midplane, coming from local viscous dissipation at the disk mid-plane, like in Shakura \& Sunayev 1973; Lynden-bell \& Pringle 1974). Figure 5 shows the distribution of the kinetic temperature for two values of $\gamma$. As $T_{k}$ will vary both in $r$ and $z$, the resulting iso-temperature surfaces will make an angle with the disk plane (case $\gamma=1.5$ ) instead of being perpendicular to it in the isothermal model $(\gamma=1)$. They will asymptotically tend to be parallel to the disk plane as the gradient is steeper in the $z$ direction.

\section{Type II}

We also generated another disk model (type II), with a different radial and vertical temperature distribution. In this model, the disk "atmosphere" $\left(z>z_{q}\right)$ follows a power law temperature distribution with radius, while the temperature in the disk midplane is constant below some radius $R_{q}$.

For $r \geq R_{q}$ :

$T_{k}(r, z)=T_{\mathrm{o}}\left(\frac{r}{100 \mathrm{AU}}\right)^{-q}$.

For $r<R_{q}$ :

$T_{k}\left(r, z>z_{q}\right)=T_{\mathrm{o}}\left(\frac{r}{100 \mathrm{AU}}\right)^{-q}=T_{\mathrm{atm}}(r)$

$T_{k}\left(r, z \leq z_{q}\right)=T_{\text {atm }}(r)$

$$
+\left(T_{\mathrm{o}}\left(\frac{R_{q}}{100 \mathrm{AU}}\right)^{-q}-T_{\mathrm{atm}}(r)\right)\left(\cos \left(\frac{\pi z}{2 z_{q}}\right)\right)^{2 \delta} .
$$

With $z_{q} \simeq 2 H$ and $\delta \approx 1-2$, this analytic form is a better representation of the temperature distribution found by dA99, provided $R_{q}$ is adjusted according to the disk mass.

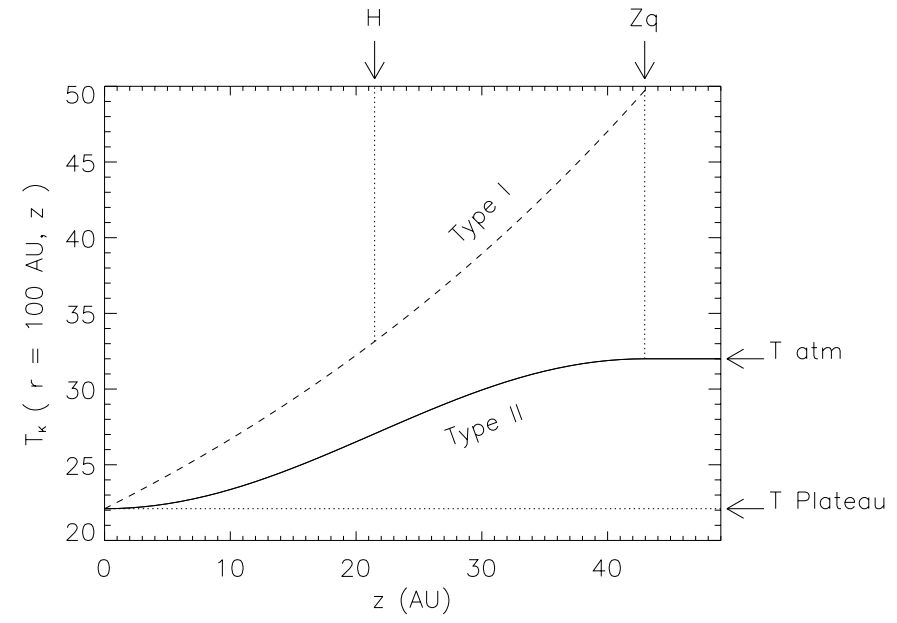

Fig. 6. Comparison of the behavior of the kinetic temperature laws for type I and type II models versus the vertical scale $z: T_{k}(r=100 \mathrm{AU}, z)$. type I: $\gamma=1.5$ (close to CG97). type II: $\delta=2, r \leq R_{q}$ with $R_{q}=$ $180 \mathrm{AU}$ (close to dA99).

Figure 6 describes the kinetic temperature laws versus $z$ for both models when $r=100 \mathrm{AU}$ ( $r \leq R_{q}$ for type II).

Note that a vertical temperature gradient can have a strong influence on the disk images in spectral lines. This can be understood in Fig. 3. With a positive gradient, at the systemic velocity, the $\mathrm{CO}$ line brightness will increase around the spatial position defined by the plane $X_{0}=0 \mathrm{AU} ; Y \sim-100 /-200 \mathrm{AU}$ (Fig. 3, frame A1) where $\mathrm{CO}$ probes scales up to a few times above the local hydrodynamic scale height.

\subsection{Influence on the derived parameters}

Before analyzing the data, we investigate how much the derived type 0 parameters are affected by the presence of a type I or type II temperature gradient. We proceed as follows. We generate disks models in ${ }^{13} \mathrm{CO}$ and ${ }^{12} \mathrm{CO} J=2 \rightarrow 1$ with vertical gradients of types I and II and minimize them assuming that there is no vertical gradient inside the disk. The aim of such minimizations is to determine what are the most important parameters that influence the minimizations and if any secure criterion can be used to constrain a gradient. Except for vertical gradients, the generated models use disk parameters relatively close to those inferred from ${ }^{12} \mathrm{CO} J=1 \rightarrow 0$ in the DM Tau disk by GD98 (see Tables 1 and 2).

Table 1 presents the test models with a type I gradient, with $\gamma=1.5$ to be consistent with CG97, and the best minimization without gradient. Note that the $\chi^{2}$ (last line) is slightly different to standard definition because there is no noise added to the model. This should be equal to "zero" if the fit of the model would perfectly represent the model itself. In other words, this $\chi^{2}$ indicates the departure of the model. The ${ }^{12} \mathrm{CO}$ and ${ }^{13} \mathrm{CO}$ analysis shows significant differences on the absolute intensity (given by $T_{\mathrm{o}}$ ) and the index of the temperature power law $(q)$. The index appears smaller for the ${ }^{12} \mathrm{CO} J=2 \rightarrow 1$ than for the ${ }^{13} \mathrm{CO} J=1 \rightarrow 0$ and for which it is close to the input value at the mid-plane. From Fig. 2, there is a relatively straightforward explanation. With the ${ }^{13} \mathrm{CO} J=1 \rightarrow 0$ isotope, we probe the disk 
Table 1. Tests on model: influence of a type I temperature gradient on the determination of the disk parameters minimized with a type 0 disk model. The abundance ratio between ${ }^{13} \mathrm{CO}$ and ${ }^{12} \mathrm{CO}$ is fixed to 70 . The parameters are normalized at 100 AU. Note that $\chi^{2}=0$ means that the fit perfectly represents the model. The varied parameters in the minimizations are in bold face.

\begin{tabular}{|c|c|c|c|c|c|}
\hline Parameter & $\begin{array}{l}\text { Type I } \\
\text { model }\end{array}$ & $\begin{array}{l}\text { minimization } \\
{ }^{13} \mathrm{CO}(1-0)\end{array}$ & $\begin{array}{l}\text { minimization } \\
{ }^{13} \mathrm{CO}(2-1)\end{array}$ & $\begin{array}{l}\text { minimization } \\
{ }^{12} \mathrm{CO}(2-1)\end{array}$ & $\begin{array}{c}\text { minimization } \\
\mathrm{C}^{18} \mathrm{O}(2-1)\end{array}$ \\
\hline$n_{\mathrm{o}}(\mathrm{CO})\left(\mathrm{cm}^{-3}\right)$ & 1200 & 1200 & 1200 & 1200 & 1200 \\
\hline$s=1.5+p-q / 2$ & 2.75 & 2.75 & 2.75 & 2.75 & 2.75 \\
\hline$T_{\mathrm{o}}(\mathrm{K})$ & 25 & 32.9 & 29.3 & 42.2 & 37 \\
\hline$q$ & 0.60 & 0.63 & 0.44 & 0.48 & 0.58 \\
\hline$v_{\mathrm{o}}\left(\mathrm{km} \mathrm{s}^{-1}\right)$ & 2.10 & 2.24 & 2.12 & 2.30 & 2.35 \\
\hline$i\left(^{\circ}\right)$ & -40 & -37.1 & -39.6 & -34.9 & -34.5 \\
\hline $\mathrm{PA}\left({ }^{\circ}\right)$ & 63 & 62.7 & 62.7 & 62.5 & 62.7 \\
\hline$R_{\text {Out }}(\mathrm{AU})$ & 650 & 643 & 648 & 643 & 610 \\
\hline$\gamma$ & 1.5 & 1 & 1 & 1 & 1 \\
\hline$\chi^{2}$ & & 2 & 9 & 490 & 4 \\
\hline
\end{tabular}

mid-plane whose temperature distribution with radius follows the same distribution as obtained with no vertical gradient. The difference appears strongly with the ${ }^{12} \mathrm{CO} J=2 \rightarrow 1$ transition. As previously seen, this transition probes a much higher height ( 200-300 AU above the plane, see Fig. 2), thus giving access to the radial variation of the temperature at this height in the disk atmosphere, which possesses a different radial behavior as can be seen in Fig. 5. Iso-temperature lines separate out with height in the disk, thus leading to a flatter radial dependence of the temperature (i.e. smaller $q$ ).

Table 2 presents the test models with a type II gradient, with $R_{q}=180 \mathrm{AU}$ and $z_{q}=2 \mathrm{H}$, which is representative of the dA99 dust temperature distribution, and the best minimization without gradient. In this case, the ${ }^{12} \mathrm{CO}$ data closely represents the outer disk atmosphere, while $\mathrm{C}^{18} \mathrm{O} J=2 \rightarrow 1$ and $\mathrm{C}^{18} \mathrm{O} J=$ $1 \rightarrow 0$ find a lower value for $q$, as expected since they probe closer to the disk mid-plane.

The minimization on type II gradient models $\left({ }^{13} \mathrm{CO} J=\right.$ $2 \rightarrow 1$ ) with type 0 model leads to an inclination less than the value in input. The type II gradient possesses a pivotal point $\left(R_{q}\right.$ in AU). The type 0 models might in fact try to compensate the differences with the type II models in opacity and surface emission by lowering the inclination in the comparison. This is because using a single power law for the temperature dependence in the entire disk, the fitted type 0 temperature in the outer part lies above the real temperature, so that the brightness is reproduced by lowering the opacity along the line of sight, obtained by lowering the inclination. In the inner part (below $R_{q}$ ), the type 0 fitted temperature is below the real temperature, and the flux is reproduced by increasing the emission area, which can only be done by lowering again the inclination. The effect will be particularly pronounced when the line opacity is about 1 at $R_{q}$, such as for example ${ }^{13} \mathrm{CO} J=2 \rightarrow 1$ or $J=1 \rightarrow 0$ in the DM Tau case. Note that we also checked the reversibility of the type II modelling by performing a $2 \times 2$ parameter minimization of a type II model using the type II model itself. The $\chi^{2}$ converge on all the input values within one $\sigma$.

\section{Data analysis}

We present in Table 3 , the $\chi^{2}$ minimization performed on 2-parameter fits with a type 0 model as described in GD98.
Table 4 summarizes the 4-parameter ${ }^{13} \mathrm{CO}$ minimization obtained with the same model. We caution that, since only 2 or 4-parameter fits were used, the error bars are underestimates of the true errors, although not by a large amount since the parameter couplings we have neglected should be small. The results of the type II minimization of the ${ }^{13} \mathrm{CO}$ data are given in Table 5.

The results are shown in Figs. 7 and 8. Note that the volume density $n_{0}(\mathrm{CO})$ and the surface density $\Sigma_{0}(\mathrm{CO})$ are always referenced to the abundance of ${ }^{12} \mathrm{CO}$, the main isotope of $\mathrm{CO}$.

\section{1. ${ }^{12} \mathrm{CO}$ data}

As shown in Figs. 2 and 3, both the ${ }^{12} \mathrm{CO} J=2 \rightarrow 1$ and $J=1 \rightarrow 0$ transitions are optically thick and almost probe the same region of the disks. The 2-parameter minimization therefore gives the same results. The results obtained on the minimization of both transitions are presented in Table 3, and are adapted from Simon et al. (2000).

\section{2. $C^{18} O J=2 \rightarrow 1$}

We also present in Table 3 the results of the best 2-parameter $\mathrm{C}^{18} \mathrm{O} J=2 \rightarrow 1$ fits. Since these observations have the lowest signal to noise, they do not bring more precise constraints on the disk parameters than the ${ }^{13} \mathrm{CO}$ results presented below. However, they indicate that the abundance ratio ${ }^{13} \mathrm{CO} / \mathrm{C}^{18} \mathrm{O}$ is compatible with the standard isotopic ratio of 70 and 550. They also confirm the large local line width, $\simeq 0.15 \mathrm{~km} \mathrm{~s}^{-1}$, found with ${ }^{13} \mathrm{CO}$ and the outer radius.

\section{3. ${ }^{13} \mathrm{CO}$ data minimization}

\section{2-parameter type 0 minimization}

Following GD98, there are a priori 14 parameters relevant for the images of the ${ }^{13} \mathrm{CO}$ line: $x_{0}, y_{0}, \mathrm{PA}, V_{\mathrm{LSR}}, V_{0}, v, i, R_{\mathrm{out}}$, $T_{0}, q, \Sigma_{0}, p, \mathrm{~d} V, X\left({ }^{13} \mathrm{CO}\right)$. PA and $V_{\mathrm{LSR}}$ are geometrical parameters which are well constrained independently of the assumed physical parameters. $x_{0}, y_{0}$ are determined from the continuum data, and $v=0.5$ for Keplerian motions. The turbulent linewidth $\mathrm{d} V$ is also fitted and appears larger than for CO lines. 
Table 2. Tests on model: influence of a type II temperature gradient on the determination of the disk parameters minimized with a type 0 disk model. The abundance ratio between ${ }^{13} \mathrm{CO}$ and ${ }^{12} \mathrm{CO}$ is fixed to 70 . The parameters are normalized at 100 AU. Note that $\chi^{2}=0$ means that the fit perfectly represents the model. The varied parameters in the minimizations are in bold face.

\begin{tabular}{|c|c|c|c|c|c|}
\hline Parameter & $\begin{array}{l}\text { Type II } \\
\text { model }\end{array}$ & $\begin{array}{l}\text { minimization } \\
{ }^{13} \mathrm{CO}(1-0)\end{array}$ & $\begin{array}{l}\text { minimization } \\
{ }^{13} \mathrm{CO}(2-1)\end{array}$ & $\begin{array}{l}\text { minimization } \\
{ }^{12} \mathrm{CO}(2-1)\end{array}$ & $\begin{array}{c}\text { minimization } \\
\mathrm{C}^{18} \mathrm{O}(2-1)\end{array}$ \\
\hline$n_{\mathrm{o}}(\mathrm{CO})\left(\mathrm{cm}^{-3}\right)$ & 1200 & 1200 & 1200 & 1200 & 1200 \\
\hline$s=1.5+p-q / 2$ & 2.75 & 2.75 & 2.75 & 2.75 & 2.75 \\
\hline$T_{\mathrm{o}}(\mathrm{K})$ & 35 & 20.6 & 25.9 & 30.2 & 28.5 \\
\hline$q$ & 0.60 & 0.27 & 0.35 & 0.51 & 0.26 \\
\hline$v_{\mathrm{o}}\left(\mathrm{km} \mathrm{s}^{-1}\right)$ & 2.10 & 2.09 & 2.19 & 2.09 & 2.30 \\
\hline$i\left(^{\mathrm{o}}\right)$ & -40 & -40.3 & -38.9 & -39.9 & -35.4 \\
\hline $\mathrm{PA}\left({ }^{\circ}\right)$ & 63 & 62.6 & 62.7 & 62.3 & 62.7 \\
\hline$R_{\text {out }}(\mathrm{AU})$ & 650 & 650.4 & 631 & 644 & 590 \\
\hline$R_{q}^{*}$ & 250 & - & - & - & - \\
\hline$z_{q}$ and $\delta^{*}$ & $2 \mathrm{H}$ and 2 & - & - & - & - \\
\hline$x^{2}$ & & 1 & 19 & 40 & 5 \\
\hline
\end{tabular}

${ }^{*} R_{q}, z_{q}$ and $\delta$ are not defined and have no meaning for the type 0 model.

The abundance appears as a scale factor for the surface density $\Sigma_{0}$, which can only be set by comparing with other mass determination (e.g. from dust emission, see Sect. 5.4). Although, in principle, the remaining parameters are all coupled, the pair $\left(V_{0} \sin i, i\right)$ should not depend significantly on temperature or density distribution (see GD98), and a separate fit was done for this pair of parameters. We further neglect the coupling between $R_{\text {out }}$ and the remaining parameters $T_{0}, q, \Sigma_{0}, p$, since it is only due to limited angular resolution.

Table 3 presents the disk parameters derived with the above procedures. The outer radius is well defined, $R_{\text {out }}=640 \pm$ $10 \mathrm{AU}$ from both ${ }^{13} \mathrm{CO}$ transitions. We note clear differences for $T_{0}$ from ${ }^{12} \mathrm{CO}$ and ${ }^{13} \mathrm{CO}, J=1 \rightarrow 0$ and $J=2 \rightarrow 1$ with values of $q$ decreasing from 0.63 to -0.3 , although the latter is only at 2 sigmas from $q=0$. We conclude from this analysis that a simple radial temperature variation seems unable to provide a satisfactory fit for all the transitions simultaneously, as expected in the case of a vertical temperature gradient does exist in the disk.

\section{4-parameter type 0 minimization}

However, the previous 2-parameter analysis arbitrarily separates $\Sigma$ and $T$, whereas these parameters are coupled because the ${ }^{13} \mathrm{CO}$ transitions involved are partly optically thin. Therefore, we investigate a global fit taking into account the presence of this coupling. The calculations presented in Sect. 3.3 show that the natural analysis to perform is a minimisation using the 4 parameters $\left\{\Sigma_{0} T_{0}, \Sigma_{0} / T_{0}, p+q, p-q\right\}$, since, in the optically thin LTE and constant abundance case, the brightness temperature for the ${ }^{13} \mathrm{CO} J=1 \rightarrow 0$ transition should depend only on $\Sigma_{0} / T_{0}$ and $p-q$, whereas the brightness dependence of the ${ }^{13} \mathrm{CO} J=2 \rightarrow 1$ transition is more complex, but mostly depend on $\left(\Sigma_{0} T_{0}\right)$ and $p+q$. The other fixed parameters are presented in Table 4. From this 4-parameter $\chi^{2}$ cube, we extract the 2-parameter $\chi^{2}$ surfaces presented in Fig. 7 for both ${ }^{13} \mathrm{CO}$ transitions observed. ${ }^{13} \mathrm{CO} J=1 \rightarrow 0$, which was expected from Sect. 3.3 to constrain essentially $p-q$, in fact allows a broad range of values for $(p, q) .{ }^{13} \mathrm{CO} J=2 \rightarrow 1$, being thicker, constrains $p+q$. In the $\left\{\Sigma_{0} T_{0}, \Sigma_{0} / T_{0}\right\} \chi^{2}$ planes, we overplotted the slopes corresponding to constant excitation temperatures of 10,20 and $30 \mathrm{~K}$. A constant temperature of $\simeq 13 \mathrm{~K}$ provides an appropriate fit to the ${ }^{13} \mathrm{CO} J=1 \rightarrow 0$ data, while temperatures of order $20 \mathrm{~K}$ are required for ${ }^{13} \mathrm{CO} J=$ $2 \rightarrow 1$. Hence, the left and middle panels of Fig. 7 indicate that different temperatures are required to represent both lines. This is emphasized in the rightmost panels, which present the projected $\chi^{2}$ surfaces resulting from the combination of both transitions (note that the rightmost panels are not just the sum of the left and middle ones since all 4 parameters are coupled). The best combination lies near the 4 to $6 \sigma$ contours from the best independent solutions.

\section{4-parameter type II minimization}

The ${ }^{12} \mathrm{CO}$ and ${ }^{13} \mathrm{CO}$ type 0 analysis presented above clearly demonstrates that a simple power law for the temperature cannot fit all transitions. Since, following Sect. 3, each line is sensitive to a different depth in the disk, the increase in temperature found from ${ }^{13} \mathrm{CO} J=1 \rightarrow 0$ to ${ }^{13} \mathrm{CO} J=2 \rightarrow 1$ and ${ }^{12} \mathrm{CO}$ suggest a vertical temperature gradient with temperatures above the disk plane.

We therefore decided to go deeper in the analysis by performing a type II minimization, which explicitely takes into account the presence of a vertical gradient in the inner disk. Following the description of this model in Sect. 4.1, it implies minimization on the four coupled parameters $\left\{R_{q}, z_{q} / H\right.$, $\left.n_{0}(\mathrm{CO}), s\right\} . R_{q}$ defines the articulation point of the law, at which there is a separation between the vertically isothermal behavior and the beginning of a temperature vertical gradient. $z_{q} / H$ defines the steepness of the gradient as compared to the local hydrostatic scale height. $n_{0}(\mathrm{CO})$ defines the absolute $\mathrm{CO}$ volume density value at the reference point (100 AU) and $s$ its radial variation. All these parameters fully describe a type II 
Table 3. 2-parameter analysis: best fits for $\mathrm{C}^{18} \mathrm{O},{ }^{13} \mathrm{CO}$ and ${ }^{12} \mathrm{CO}$ data $(D=140 \mathrm{pc})$. Minimization done with the type 0.

\begin{tabular}{lccccc}
\hline \hline Parameter & minimization & minimization & minimization & minimization & minimization \\
& $\mathrm{C}^{18} \mathrm{O}(2-1)$ & ${ }^{13} \mathrm{CO}(2-1)$ & ${ }^{13} \mathrm{CO}(1-0)$ & ${ }^{12} \mathrm{CO}(2-1)$ & ${ }^{12} \mathrm{CO}(1-0)$ \\
\hline$n_{\mathrm{o}}(\mathrm{CO})\left(\mathrm{cm}^{-3}\right)$ & 1400 & $\mathbf{1 4 0 0} \pm \mathbf{1 2 0}$ & $\mathbf{1 8 0 0} \pm \mathbf{2 0 0}$ & 1400 & 1400 \\
$s=1.5+p-q / 2$ & $\mathbf{2 . 7 5} \pm \mathbf{0 . 0 5}$ & $\mathbf{2 . 7 5} \pm \mathbf{0 . 1 0}$ & $\mathbf{2 . 7} \pm \mathbf{0 . 1}$ & 2.75 & 2.75 \\
$T_{\mathrm{o}}(\mathrm{K})$ & $\mathbf{3 5} \pm \mathbf{3}$ & $\mathbf{2 2} \pm \mathbf{1}$ & $\mathbf{1 4} \pm \mathbf{2}$ & $\mathbf{3 2 . 5} \pm \mathbf{0 . 5}$ & $\mathbf{3 1} \pm \mathbf{2}$ \\
$q$ & $\mathbf{0 . 3 0} \pm \mathbf{0 . 1 0}$ & $\mathbf{0 . 3 0} \pm \mathbf{0 . 0 5}$ & $\mathbf{- 0 . 3 0} \pm \mathbf{0 . 1 5}$ & $\mathbf{0 . 6 3} \pm \mathbf{0 . 0 1}$ & $\mathbf{0 . 6 3} \pm \mathbf{0 . 0 5}$ \\
$v_{\mathrm{o}}\left(\mathrm{km} \mathrm{s}^{-1}\right)$ & $\mathbf{2 . 1 5} \pm \mathbf{0 . 1 0}$ & $\mathbf{2 . 2 5} \pm \mathbf{0 . 0 5}$ & $\mathbf{2 . 0 1} \pm \mathbf{0 . 1 0}$ & $\mathbf{2 . 1 8} \pm \mathbf{0 . 1 0}$ & $\mathbf{2 . 1 5} \pm \mathbf{0 . 2 0}$ \\
$i\left({ }^{(}\right)$ & $-\mathbf{3 4} \pm \mathbf{3}$ & $-\mathbf{3 1} \pm \mathbf{1}$ & $-\mathbf{3 8} \pm \mathbf{2}$ & $-\mathbf{3 2} \pm \mathbf{2}$ & $\mathbf{3 3} \pm \mathbf{2}^{*}$ \\
$\mathrm{PA}\left({ }^{\circ}\right)$ & $\mathbf{6 6} \pm \mathbf{2}$ & $\mathbf{6 5} \pm \mathbf{1}$ & $\mathbf{6 5} \pm \mathbf{1}$ & $\mathbf{6 7} \pm \mathbf{1}$ & $\mathbf{6 6} \pm \mathbf{2}$ \\
$V_{\mathrm{LSR}}\left(\mathrm{km} \mathrm{s}^{-1}\right)$ & $\mathbf{6 . 0 1} \pm \mathbf{0 . 0 2}$ & $\mathbf{6 . 0 3} \pm \mathbf{0 . 0 1}$ & $\mathbf{6 . 0 9} \pm \mathbf{0 . 0 1}$ & $\mathbf{6 . 0 5} \pm \mathbf{0 . 0 1}$ & $\mathbf{6 . 0 5} \pm \mathbf{0 . 0 2}$ \\
$R_{\text {out }}(\mathrm{AU})$ & $\mathbf{6 0 0} \pm \mathbf{5 0}$ & $\mathbf{6 4 0} \pm \mathbf{1 5}$ & $\mathbf{6 5 0} \pm \mathbf{2 0}$ & $\mathbf{8 0 0} \pm \mathbf{5}$ & $\mathbf{7 9 0} \pm \mathbf{2 0}$ \\
$\mathrm{d} V\left(\mathrm{~km} \mathrm{~s}^{-1}\right)$ & $\mathbf{0 . 1 4} \pm \mathbf{0 . 0 3}$ & $\mathbf{0 . 1 6} \pm \mathbf{0 . 0 1}$ & $\mathbf{0 . 1 6} \pm \mathbf{0 . 0 2}$ & $\mathbf{0 . 0 7} \pm \mathbf{0 . 0 1}$ & $\mathbf{0 . 0 7} \pm \mathbf{0 . 0 2}$ \\
\hline$\gamma$ & 1. & 1. & 1. & 1. & 1. \\
\hline
\end{tabular}

Parameters with no error bars are adopted, the varied parameters are in bold faces.

* See Simon et al. (2000) for the interpretation of the sign of the inclination.
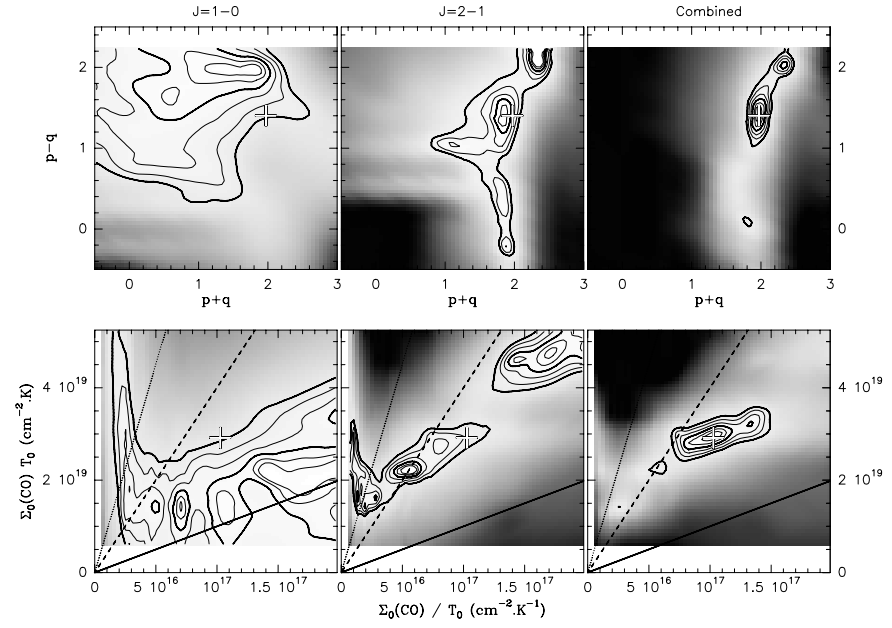

Fig. 7. Analysis with the type 0 model (top) $\chi^{2}$ surface as function of $p+q, p-q$ for the $J=1-0, J=2-1$ data and the combined $\chi^{2}$. (bottom) $\chi^{2}$ surface as function of $\Sigma / T, \Sigma T$ for the $J=1-0, J=2-1$ data and the combined $\chi^{2}$. Contours are spaced by $1 \sigma$, bold contours are at 3 and $6 \sigma$. The overplotted slopes correspond to excitation temperatures of $10 \mathrm{~K}$ (thick line), $20 \mathrm{~K}$ (dashed line) and $30 \mathrm{~K}$ (thin line). A cross shows the location of the minimum found for the combined $\chi^{2}$. Referenced to the abundance of ${ }^{12} \mathrm{CO}$.

model. $R_{q}$ and $z_{q}$ are fully defined in Sect. 4.1, type II model, whereas $n_{0}(\mathrm{CO}), s$ and $H$ are defined in the Appendix. We use $T_{0}=32 \mathrm{~K}$, and $q=0.63$, the law derived from the observation of the "CO atmosphere" of the disk with the ${ }^{12} \mathrm{CO}$ transitions, and all other parameters were taken from the type 0 model fit. The ${ }^{13} \mathrm{CO} J=1 \rightarrow 0\left\{R_{q}, z_{q} / H\right\} \chi^{2}$ plane indicates a rather well defined value of $R_{q} \simeq 460 \pm 30 \mathrm{AU}$, but high values of $z_{q} / H\left(z_{q} / H \geq 6\right)$. Since the bulk of the ${ }^{13} \mathrm{CO} J=1 \rightarrow 0$ emission is from low values of $z / H$, this is consistent with a constant temperature; this temperature is defined by $R_{q}: T_{k}\left(R_{q}\right)=T_{0} \times\left(R_{q} / 100\right)^{-q} \sim 12-13 \mathrm{~K}$. As expected, these results are in complete agreement with those of Fig. 7. The ${ }^{13} \mathrm{CO} J=2 \rightarrow 1$ line, which traces slightly upper layers in the disk can better probe the vertical gradient and constrains

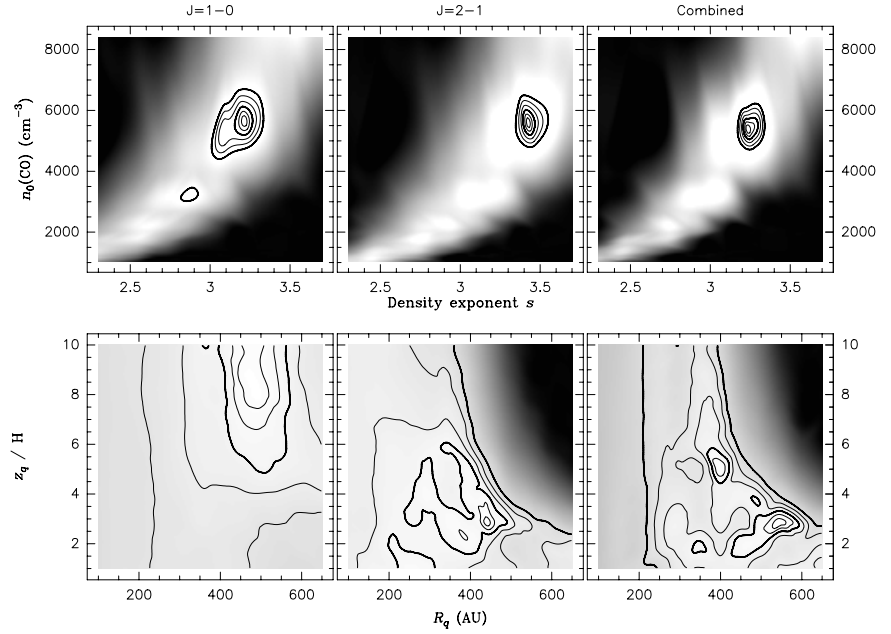

Fig. 8. Analysis with type II model (top) $\chi^{2}$ surface as function of $f, s$ for the ${ }^{13} \mathrm{CO} J=1 \rightarrow 0$ and $J=2 \rightarrow 1$ data. (bottom) $\chi^{2}$ surface as function of $R_{q}, z_{q} / \mathrm{H}$ for the ${ }^{13} \mathrm{CO} J=1 \rightarrow 0$ and $J=2 \rightarrow 1$ data. Contours are spaced by $1 \sigma$, bold contours are at 3 and $6 \sigma$. In this model, $\Sigma_{0}(\mathrm{CO})$ can be derived from $n_{0}(\mathrm{CO})$, assuming a scale height of $H \simeq 10 \mathrm{AU}$ at $100 \mathrm{AU}: \Sigma_{0}(\mathrm{CO}) \sim 1.2 \times 10^{18} \mathrm{~cm}^{-2}$. Referenced to ${ }^{12} \mathrm{CO}$ abundance.

$z_{q} / H$. The combined $\left\{R_{q}, z_{q} / H\right\} \chi^{2}$ plane allows a common solution to be found within $3 \sigma$ of each individual fit. This is more consistent than in the type 0 model, but still indicates that the type II model is not a perfect representation of the disk structure (in temperature and/or density). The agreement with the data could be improved by using a hotter, steeper temperature law $T_{k}\left(r, z>z_{q}\right)$, as suggested by the results of Table 2 , since it appears that even ${ }^{12} \mathrm{CO}$ does not exactly represent the upper layers $\left(z>z_{q}\right)$.

\subsection{Continuum as an indicator of the $\mathrm{H}_{2}$ disk mass}

A detailed analysis of the continuum data is beyond the scope of this paper. However, it is important to note at this stage how we use the dust continuum emission in order to determine the 
Table 4. 4-parameter analysis: adopted values for the parameters which do not vary for the ${ }^{13} \mathrm{CO}$ data minimization done with the type 0 model. We use a distance of $D=140 \mathrm{pc}$.

\begin{tabular}{lc}
\hline \hline Parameter & Type 0 \\
\hline$v_{\mathrm{o}}\left(\mathrm{km} \mathrm{s}^{-1}\right)$ & 2.03 \\
$i\left(\left(^{\circ}\right)\right.$ & -37 \\
$\mathrm{PA}\left({ }^{\circ}\right)$ & 66 \\
$V_{\mathrm{LSR}}\left(\mathrm{km} \mathrm{s}^{-1}\right)$ & 6.05 \\
$R_{\text {out }}(\mathrm{AU})$ & 650 \\
$\mathrm{~d} V\left(\mathrm{~km} \mathrm{~s}^{-1}\right)$ & 0.15 \\
\hline
\end{tabular}

Table 5. 4-parameter analysis: adopted values for the parameters which do not vary for the ${ }^{13} \mathrm{CO}$ data minimization done with the type II model. We use a distance of $D=140 \mathrm{pc}$.

\begin{tabular}{lc}
\hline \hline Parameter & Type II \\
\hline$T_{\mathrm{o}}(\mathrm{K})=T_{\text {atm }}(100 \mathrm{AU})$ & 32 \\
$q$ & 0.63 \\
$v_{\mathrm{o}}\left(\mathrm{km} \mathrm{s}^{-1}\right)$ & 2.03 \\
$i\left(^{\circ}\right)$ & -37 \\
$\mathrm{PA}\left({ }^{\circ}\right)$ & 66 \\
$V_{\mathrm{LSR}}\left(\mathrm{km} \mathrm{s}^{-1}\right)$ & 6.05 \\
$R_{\text {out }}(\mathrm{AU})$ & 650 \\
$\mathrm{~d} V\left(\mathrm{~km} \mathrm{~s}^{-1}\right)$ & 0.15 \\
\hline
\end{tabular}

$\mathrm{CO}$ depletion factor because the disk mass is best estimated from the mostly optically thin continuum flux at $1.3 \mathrm{~mm}$.

Following Beckwith et al. (1990), we assume for the absorption coefficient $\kappa_{v}=0.1 \times\left(v / 10^{12} \mathrm{~Hz}\right)^{\beta}\left(\mathrm{cm}^{2} \mathrm{~g}^{-1}\right)$. For DM Tau, we use $\beta=0.8$ because this value gives the best fits of the continuum interferometric maps. It leads to $\kappa_{220}=0.030$ and $\kappa_{110}=0.017 \mathrm{~cm}^{2} \mathrm{~g}^{-1}$. This dust absorption coefficient takes into account the gas by assuming a gas-to-dust ratio of $Z_{\mathrm{d}}=100$ and the mass is the total dust+gas mass of the disk. Using this dust absorption coefficient, we fit the continuum emission at all wavelengths. Taking $\beta \simeq 0.8$, we performed a 4-parameter $\chi^{2}$ minimization on $\left\{p+q, p-q, \Sigma_{0} T_{0}, \Sigma_{0} / T_{0}\right\}$ as described in Pietu et al. (2002). As expected, since the dust emission is mostly optically thin, the minimization contrains $\left(\Sigma_{0}\left(\mathrm{H}_{2}\right) T_{0}\right)_{\text {dust }} \sim 3 \times 10^{24} \mathrm{~cm}^{-2} \mathrm{~K}$, and $p+q \simeq 2$. These values will be used in Sect. 6 where we discuss the density distribution and the $\mathrm{CO}$ depletion. The disk mass can then be estimated provided $T_{0}$ and $q$ are known. Because of the evidence for vertical temperature gradients, the appropriate values for $T_{0}, q$ are however unclear.

If the dust and $\mathrm{CO}$ sample the same region, the $\mathrm{CO}$ abundance is directly given by:

$X(\mathrm{CO})=\left(\Sigma_{0}(\mathrm{CO}) T_{0}\right) /\left(\Sigma_{0}\left(\mathrm{H}_{2}\right) T_{0}\right)_{\mathrm{dust}}$

and the $\mathrm{CO}$ "depletion factor" is given by the ratio $f(\mathrm{CO})=$ $X_{\text {ref }} / X(\mathrm{CO})$, where $X_{\text {ref }}$ is the abundance in the Taurus molecular cloud. We use $X_{\text {ref }}=7 \times 10^{-5}$ for ${ }^{12} \mathrm{CO}, 1.6 \times 10^{-6}$ for ${ }^{13} \mathrm{CO}$, and $1.3 \times 10^{-7}$ for $\mathrm{C}^{18} \mathrm{O}$ respectively (corresponding to isotopic ratios for 70 and 550, Langer \& Penzias 1993). It is important to note that such a "depletion factor", although defined by reference to a standard $\mathrm{CO}$ abundance, also depends on the dust properties and the gas-to-solid ratio $\left(f(\mathrm{CO}) \propto X_{\text {ref }} / X(\mathrm{CO}) / \kappa_{v}\right)$. It also relies on the assumption of equal dust and CO temperatures. Such a depletion factor cannot be therefore simply considered as a $\mathrm{CO}$ depletion with respect to standard $\mathrm{CO}$ abundances encountered in the Taurus clouds.

To avoid using these extra parameters, we have chosen to present all $\mathrm{CO}$ results in the measured quantity which is the ${ }^{12} \mathrm{CO}$ surface density $\Sigma\left({ }^{12} \mathrm{CO}\right)$ (or the volume density $n\left({ }^{12} \mathrm{CO}\right)$ ), using the standard isotopomer ratios of 70 and 550 to convert from ${ }^{13} \mathrm{CO}$ and $\mathrm{C}^{18} \mathrm{O}$ measurements.

\section{Discussion}

\subsection{Line widths: Warp or turbulence}

We note that the non-thermal component of the linewidth, $\mathrm{d} V$, increases from $\mathrm{d} V=0.07 \pm 0.02 \mathrm{~km} \mathrm{~s}^{-1}$ measured with ${ }^{12} \mathrm{CO}$ to values around $0.15 \pm 0.02 \mathrm{~km} \mathrm{~s}^{-1}$ from the ${ }^{13} \mathrm{CO}$ and $\mathrm{C}^{18} \mathrm{O}$ isotopes. A straightforward interpretation would be that the turbulence is higher in the disk mid-plane, sampled by the $\mathrm{CO}$ isotopomers, than in the disk atmosphere, sampled by ${ }^{12} \mathrm{CO}$.

However, Table 3 also shows that the derived disk inclination differs from one isotopomer to the other. Higher inclinations are found from the rarer isotopomers than from ${ }^{12} \mathrm{CO}$. Systematic bias resulting from the analysis by a type 0 model of a more complex disk structure would result in the opposite effect (lower inclination for ${ }^{13} \mathrm{CO}$ and $\mathrm{C}^{18} \mathrm{O}$, see Sect. 4.2), suggesting that the difference in inclination is real. Since ${ }^{13} \mathrm{CO}$ and $\mathrm{C}^{18} \mathrm{O}$ preferentially sample the inner part of the disk $(r \leq 200 \mathrm{AU})$, while ${ }^{12} \mathrm{CO}$ is sensitive to the whole disk $(r \simeq$ $800 \mathrm{AU})$, this could be indicative of a warped disk. Continuum emission also provides a measurement of the inner disk inclination: a value of $36 \pm 4^{\circ}$ is found from the $1.4 \mathrm{~mm}$ measurements. Unfortunately, this is only a lower limit, since it should be corrected from seeing effects. If the disk is warped, the change of inclination with radius will result in enhanced projected velocity gradients, which will mimic an increase in linewidth near the disk center. The magnitude of the effect depends on the warp intensity, and is difficult to estimate. However, one should remember that the apparent inclinations we measure are lower limits to the inclination variations, since they are averaged values over large areas of the disk.

Note that inclination and line widths are well de-coupled parameters. Inclination is determined from the shape of the individual channel images, while the line width is constrained by the total flux (see Beckwith \& Sargent 1993; Guilloteau \& Dutrey 1994, 1998). Hence, with the present observations, the two alternatives (warp or enhanced turbulence in the disk plane) remain plausible.

\subsection{Dynamical mass}

Analysing the data with an inappropriate model could lead to inconsistent inclination determination, and hence to errors on the stellar mass derived from Keplerian motions. Table 3 shows some possible effects at the level of a few degrees, leading to 
errors of $\sim 9 \%$ on the stellar mass, but with $\mathrm{C}^{18} \mathrm{O}$ and ${ }^{13} \mathrm{CO} J=$ $1 \rightarrow 0$ data being the least affected. These errors are at most comparable to the $1 \sigma$ error on real data.

\subsection{Possible selective photodissociation of $\mathrm{CO}$}

The observed outer radius of DM Tau, in the ${ }^{13} \mathrm{CO} J=1 \rightarrow 0$ and $J=2 \rightarrow 1$ transitions ( $\sim 640 \mathrm{AU}$ ) is significatively different from the ${ }^{12} \mathrm{CO} J=1 \rightarrow 0$ and $J=2 \rightarrow 1$ ones ( $\left.~ 800 \mathrm{AU}\right)$. The ${ }^{12} \mathrm{CO}$ lines are optically thick. The observed radius in these transitions reveal a clear cut off in the radial density distribution, whereas the ${ }^{13} \mathrm{CO}$ outer radius could be governed by its ability to photodissociate at higher extinction than its main isotope.

The behaviour of the photodissociation rates for both $\mathrm{CO}$ isotopes as a result of self-shielding, mutual shielding, other atoms and molecules absorption (mainly $\mathrm{H}$ and $\mathrm{H}_{2}$ ) as well as dust screening are discussed in the interstellar cloud cases by e.g. van Dishoeck \& Black (1988) and Glassgold et al. (1985).

If we look at the Fig. 8 of van Dishoeck \& Black (1988), we see that the photodissociation of $\mathrm{CO}$ becomes effective as soon as the $\mathrm{H}_{2}$ column density is lower than $1.4 \times 10^{21} \mathrm{~cm}^{-2}$, for a model with $I_{\mathrm{UV}} \approx 1$, i.e. a normal interstellar radiation field (Draine 1974). In the DM Tau model of GD98, at $800 \mathrm{AU}$, the vertical column density above the disk mid-plane $N$ is given by $\frac{\sqrt{\pi}}{2} \times H \times N(r=800 \mathrm{AU}, z=0)$, i.e. $n_{\mathrm{H}_{2}} \approx 1.3 \times 10^{21} \mathrm{~cm}^{-2}$, consistent with the fact this outer radius is governed by photodissociation.

We now use the relation $N_{\mathrm{H}} / A_{\mathrm{V}}=1.59 \times 10^{21} \mathrm{~cm}^{-2} \mathrm{mag}^{-1}$ to determine the minimum $A_{\mathrm{Vth}}$ required to protect ${ }^{12} \mathrm{CO}$, which leads to $A_{\mathrm{Vth}} \approx 0.8$. The corresponding photodissociation rate at which ${ }^{12} \mathrm{CO}$ is destroyed, using the Fig. 5 of van Dishoeck \& Black (1998) is of $10^{-13} \mathrm{~s}^{-1}$. Assuming we need the same rate to dissociate ${ }^{13} \mathrm{CO}$, the corresponding $A_{\mathrm{V}}$ equals 1.15 . The column density at the ${ }^{13} \mathrm{CO}$ outer radius (640 AU) should therefore be 1.4 times the one at $800 \mathrm{AU}$. As the surface density law is given by $\Sigma(r)=\Sigma_{0} \times(r / 100 \mathrm{AU})^{-p}$, we can derive that:

$p=\frac{\log \left(\frac{\sum(640 \mathrm{AU})}{\Sigma(800 \mathrm{AU})}\right)}{\log \left(\frac{800}{640}\right)}=\frac{\log (1.4)}{\log (1.25)} \approx 1.5$.

As pointed out by van Dishoeck \& Black (1988), the CO line widths being small as compared to the natural width of most lines, it should not affect the photodissociation rates and these models provide a good comparison to the case we are studying.

However, if we now take into account that in the T Tauri disks the grain coagulation and growth has started, we expect the $N_{\mathrm{H}} / A_{\mathrm{V}}$ ratio to be higher than in molecular clouds. The shielding by dust will become less important. It should not have a strong influence on the ${ }^{12} \mathrm{CO}$ shielding as it is already effective at low dust extinction (see Fig. 5a of van Dishoeck \& Black 1988 and Fig. 3 of Glassgold et al. 1985). However, it should have an influence on the ${ }^{13} \mathrm{CO}$ photodissociation rates, which should raise. If we proceed in the same way as described above to determine the difference in $\mathrm{A}_{\mathrm{Vth}}$ required to protect ${ }^{12} \mathrm{CO}$ and ${ }^{13} \mathrm{CO}$, it implies that $\Sigma(640 \mathrm{AU}) / \Sigma(800 \mathrm{AU}) \geq 1.4$.

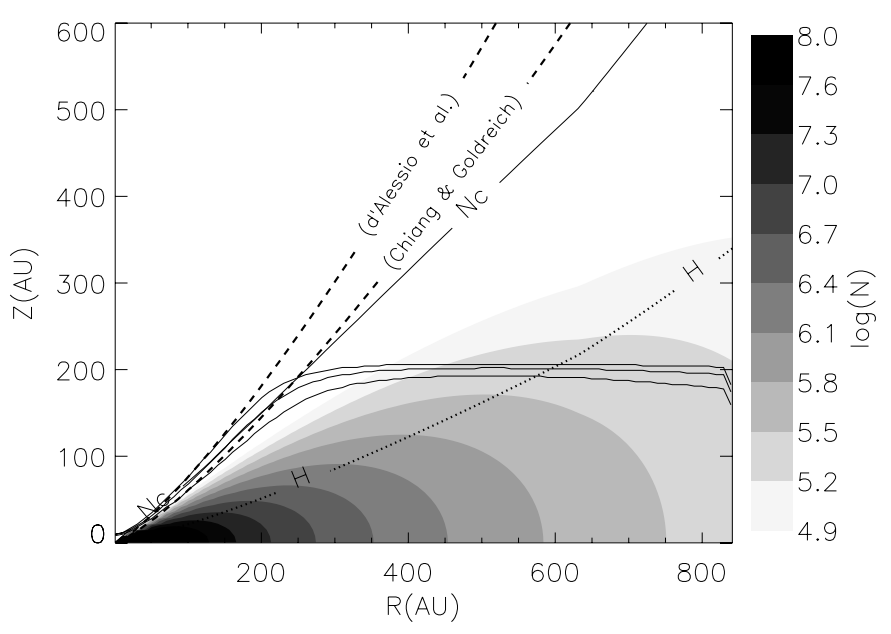

Fig. 9. Representation of the characteristic vertical scales and number densities in the DM Tau disk. The short dashed line represent the local hydrostatic scale height. The heights probed with the ${ }^{12} \mathrm{CO} J=2 \rightarrow 1$ transition with $\tau=0.5,1$ and 2 are given by the dot-dashed curves. The critical density $N_{\mathrm{c}}$ to reach LTE with this transition is given by the continuous line and shows CO is thermalized. Typical heights associated with the direct heating by stellar photons impinging on the dust (so-called "super-heated layer") are outlined by the long dashed curves in two different models. For the definition of the local hydrostatic scale height $H$, see the appendix.

Given that the uncertainty in the derivation of the observed $R_{\text {out }}$ is of $\sim 20 \mathrm{AU}$, and assuming the $A_{\mathrm{Vth}}$ ratio determination is accurate at the $10 \%$ level, it is compatible with $p \geq 0.9$. This does not provide a strong constraint on the $p$ index, but rather demonstrate that the photodissociation of $\mathrm{CO}$ is a reasonable process to explain the observations.

\subsection{Temperature}

The temperature structure of protoplanetary disks has been modeled by CG97 and dA99, in view of analyzing the IR emission from disks. Both models concentrate on the dust temperature, while we are interested in the gas temperature. However, at the densities $\left(>10^{6} \mathrm{~cm}^{-3}\right)$ and temperatures $(10-50 \mathrm{~K})$ where the $\mathrm{CO}$ emission arises, the gas cooling is very inefficient, so that the kinetic temperature is essentially identical to the dust temperature. On the other hand, the dust-gas coupling has negligible effect on the dust temperature, which is completely dominated by heating by the stellar light.

This results in two major effects on the temperature structure of the disk. The disk photosphere, defined as the surface where the optical depth of the dust to the stellar light is about unity is heated to high temperatures by stellar photons directly impinging on the disk surface. For T Tauri stars, it corresponds to $\tau_{\text {dust }}(I R) \simeq 1$. This warm "super-heated" layer reradiates at longer wavelengths to heat the disk interior.

To compare with models of super-heated layers, it is important to note that our definition of the hydrostatic scale height differs from that used in the models of CG97 and dA99 by $\sqrt{2}$, our scale height being larger (see Appendix for the details). Following our definition, the dust photosphere lies approximately at $h_{\text {phot }} \approx 4 H / \sqrt{2}$ in CG97. At the radius of $100 \mathrm{AU}$, the 


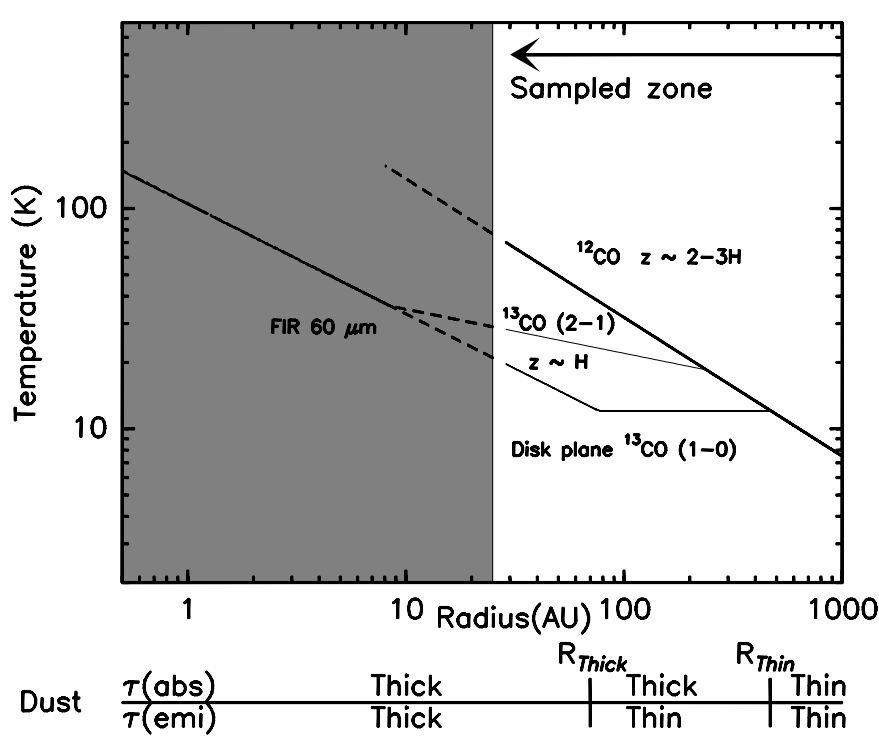

Fig. 10. Schematic representation of the gas and dust temperature in the disk mid-plane and disk atmosphere as probed with the ${ }^{13} \mathrm{CO}$ and ${ }^{12} \mathrm{CO}$ millimeter transitions. The estimate of the inner disk temperature distribution inferred from Far Infrared measurement is also reported (dotted line). The inner and outer boundary of the nearly constant temperature region are labeled $R_{\text {Thick }}$ and $R_{\text {Thin }}\left(R_{\text {Thin }}=R_{q}\right.$ in the formal description of type II model) respectively. The arrow delineates the part of the disk constrained by the PdBI observations.

midplane temperature is of $20 \mathrm{~K}$, whereas the surface is heated at $\sim 90 \mathrm{~K}$. In the model developped by dA99, $h_{\text {phot }} \approx 5 H / \sqrt{2}$, and the temperature are of $15 \mathrm{~K}$ and $60 \mathrm{~K}$ at mid-plane and dust surface, respectively. Both models assume a $0.5 M_{\odot}$ central star, with an effective temperature $T_{*}=4000 \mathrm{~K}$. The former takes $R_{*}=2.5 R_{\odot}$, whereas for the later $R_{*}=2 R_{\odot}$. Figure 9 summarizes the scale heights relevant for the comparison.

In addition to this "super-heated" layer, there is a temperature "plateau" in the disk mid-plane, resulting from a temperature gradient between the disk atmosphere and the disk plane. When the disk is optically thick to the illuminating radiation, but thin to its own emission, the disk plane is cooler than the disk atmosphere. This can happen in a well defined region of the disk with the appropriate surface density (see e.g. dA99, their Fig. 3). Closer to the star, when the surface density is higher, the mid plane temperature rises again with $q=0.5$. At the outer edge, the mid-plane temperature asymptotically reaches the temperature of the disk atmosphere. Hence, in the disk plane, as a function of radius, there is a region where the temperature law flattens, while $q$ becomes again of order 0.6 at larger distances.

Since, as shown in Fig. 9, the ${ }^{12} \mathrm{CO} J=2 \rightarrow 1$ line probe a slightly lower layer than the dust photosphere, we cannot fully constrain the "super-heated" layer from our observations. On the other hand, the temperature "plateau" can be traced by the ${ }^{13} \mathrm{CO}$ transitions.

The kinetic temperature distribution we deduce from the CO data in the DM Tau outer disk is shown Fig. 10. While $q=0.63 \pm 0.04$ from ${ }^{12} \mathrm{CO} J=1-0$ and $J=2-1$ data, with $T_{0}=$ $32 \pm 2 \mathrm{~K}$ at $100 \mathrm{AU}$, the ${ }^{13} \mathrm{CO}$ observations suggest lower values for both $q(\simeq 0-0.4)$ and $T_{0}(13 \mathrm{~K})$, although the later value depends on the assumption that the same density power law regime holds between $100 \mathrm{AU}$ (sampled by $J=1-0$ line) and 300 AU (sampled by the $J=2-1$ line). The temperature law is thus steeper above the plane than near the disk mid-plane.

The outer edge of the "plateau" $\left(R_{\text {Thin }}\right.$ in Fig. 10$)$ is determined from our data by the intersection of the $T_{\mathrm{atm}}=32 \mathrm{~K}$, $q=0.63$ law with the $T_{\text {plane }} \simeq 13 \mathrm{~K}, q \approx 0$, i.e. about $400 \mathrm{AU}$. The inner edge cannot be determined directly by our observations, due to the lack of angular resolution. However, it can be reasonably estimated by assuming the dust and gas temperature are equal. The dust temperature distribution in the inner regions can be determined from the FIR flux densities around $60 \mu \mathrm{m}$. Beckwith et al. (1990) find for DM Tau $T_{k}(1 \mathrm{AU}) \approx 110 \mathrm{~K}$ and $q \approx 0.51$ from the IRAS data. This temperature law intersects that of the "plateau" around $R_{\text {Thick }} \approx 70 \mathrm{AU}$. The direct observation of a temperature "plateau" allows to resolve the apparent inconsistencies between the low temperatures derived from the mid and FIR and the higher values derived from ${ }^{12} \mathrm{CO}$ observations at larger radius. The measurement of the inner radius would require better angular resolution at $\mathrm{mm}$ wavelengths (e.g. ALMA).

\subsection{Depletion}

Using the results on the continuum and the $\mathrm{CO}$ lines, we can estimate $X(\mathrm{CO})$ and the $\mathrm{CO}$ depletion factor $f(\mathrm{CO})$. The $\mathrm{CO}$ abundance is given by the ratio (see Fig. 7 for the values)

$$
X(\mathrm{CO})=\frac{\left(\Sigma_{0} T_{0}\right)(\mathrm{CO})}{\left(\Sigma_{0} T_{0}\right)_{\mathrm{dust}}\left(\mathrm{H}_{2}\right)}=\frac{2 \times 10^{19}}{3 \times 10^{24}} \simeq 6.6 \times 10^{-6}
$$

For $X_{\text {ref }}=7 \times 10^{-5}$, we get $f(\mathrm{CO}) \sim 10$. If we take into account the uncertainties at $3 \sigma$ on $\left(\Sigma_{0} T_{0}\right)(\mathrm{CO})$ and $\left(\Sigma_{0} T_{0}\right)_{\text {dust }}, f(\mathrm{CO})$ is ranging from $\sim 7$ to 15 . Assuming a depletion of 10 , as a mean value over the disk, is therefore reasonable.

In the type II model, we determine $n_{0}(\mathrm{CO}) \simeq 4200 \mathrm{~cm}^{-3}$, from which, using a scale height of $H \simeq 10 \mathrm{AU}$ at $100 \mathrm{AU}$, consistent with the $T_{0}=13 \mathrm{~K}$ mid-plane temperature, gives $\Sigma_{0}(\mathrm{CO}) T_{0} \simeq 2.2 \times 10^{19} \mathrm{~cm}^{2} \mathrm{~K}$, as for the type 0 models.

Using standard isotopic abundances, we derive identical depletion factors from ${ }^{13} \mathrm{CO} J=2 \rightarrow 1$ and the optically thinner transition $\mathrm{C}^{18} \mathrm{O} J=2 \rightarrow 1$, although they do not sample the same volume in the disk. This suggests that the depletion does not vary by huge factors within the disk. Note that the depletion is not sufficient to significantly deplete $\mathrm{CO}$, which is optically thick up to $800 \mathrm{AU}$, whereas the grain temperature is below the freezing point at radii above $200 \mathrm{AU}$. Except for the external region, which can be affected by other effects like photodissociation, this high opacity is ubiquitous in observed disks (GG Tau, Dutrey et al. 1994; GM Auriga, Dutrey et al. 1998; Simon et al. 2000). Hence, there is still a sufficient amount of $\mathrm{CO}$ at the temperature of the "plateau" $(13 \mathrm{~K})$ even if it is well below the $\mathrm{CO}$ freeze out point.

\subsection{Density}

The existence of a temperature "plateau" can place indirect constraints on the disk mass, because the "plateau" appears 
within a defined surface density range. In the dA99 models, this temperature plateau happens when $\Sigma_{\mathrm{d}}$ is about $0.1 \mathrm{~g} \mathrm{~cm}^{-2}$. When the disk becomes more massive (see Fig. 3 dA99), the opacity is such that the plateau never exist. The observation of a plateau therefore sets an upper limit on the disk mass. However, the precise position of this plateau depends both on the slope and the absolute value of the dust opacity function between 50 and $300 \mu \mathrm{m}$, related to the temperature range of reemission concerned (10-60 K).

With the dust properties adopted by dA99 (Draine \& Lee (1984) optical constants and Mathis et al. (1977) size distribution), the existence of the "plateau" implies $M_{\text {disk }}<4 \times$ $10^{-3} M_{\odot}$. However, this combination of dust opacity and disk mass fails to reproduce the observed mm flux densities by a factor $\simeq 10$. The discrepancy can be resolved if significant grain growth has occurred. In this case, the millimeter opacities are increased, but the shorter wavelengths opacities decrease (see for example Kruegel \& Siebenmorgen 1994, their Fig. 12), thereby moving inward the position of the plateau for a given disk mass. From $\Sigma_{0} T_{0}$ derived from the dust, and using $T_{0} \simeq 15 \mathrm{~K}$ for the dust temperature (or equivalently, using an average depletion of 10 for $\mathrm{CO}$ relative to standard Taurus abundances), our measurement indicates $\Sigma_{0} \sim 2 \times 10^{23} \mathrm{H}_{2} \mathrm{~cm}^{-2}$ at $100 \mathrm{AU}$ or $\Sigma_{\mathrm{d}} \sim 0.8 \mathrm{~g} \mathrm{~cm}^{-2}$. Although this goes in the expected direction more detailed disk models with more appropriate dust parameters are required to predict quantitatively the position and extent of the temperature "plateau".

Finally, let us note that the density exponent, $p \simeq 1.5$, agrees with the ratio of the outer radii for ${ }^{12} \mathrm{CO}$ and ${ }^{13} \mathrm{CO}$, when the $\mathrm{CO}$ distribution is truncated by photodissociation.

\section{Summary}

Using high angular $\left(1^{\prime \prime}\right)$ resolution PdBI millimeter interferometry we have mapped the circumstellar disk of the T Tauri star DM Tau in ${ }^{12} \mathrm{CO},{ }^{13} \mathrm{CO}$ and $\mathrm{C}^{18} \mathrm{O}$ in their $J=1 \rightarrow 0$ and $J=2 \rightarrow 1$ transitions. The combination of isotopes imaging allows to sample the entire line opacity range in the disk and therefore to separate and constrain the parameters describing its physical state. The analysis of the data allow to draw several conclusions:

- The observed disk external radius is lower in $\mathrm{C}^{18} \mathrm{O}$ (600 AU) and ${ }^{13} \mathrm{CO}(640 \mathrm{AU})$ than in ${ }^{12} \mathrm{CO}(800 \mathrm{AU})$. This difference is in agreement with the selective photodissociation of $\mathrm{CO}$ isotopes.

- The vertical behavior of the temperature distribution varies with the radius. We have direct observational evidence of the presence of a positive vertical temperature gradient in the DM Tau circumstellar disk. The region concerned corresponds to the transition from the totally thick to totally thin dust disk. In this regime the midplane exhibits a "plateau" of nearly constant temperature of about $\sim 13 \mathrm{~K}$.

- The existence of such a plateau put limits on the disk mass (related to the dust opacity). Comparison with current models suggest that grain growth must have occured to explain the observations.
- The measurement of CO dynamical mass is weakly affected by the existence of the temperature gradient.

This method is likely to become more general and very powerful with the next generation interferometer ALMA. Particularly, it will allow us to probe the temperature gradient not only in the outer disk but also closer to the star, at $r \simeq 10$ $30 \mathrm{AU}$, a domain which is currently impossible to study due to limited sensitivity and resolution.

Acknowledgements. We acknowledge all the PdBI IRAM staff for their help during the observations. Particular thanks to D. Nürnberger who pointed out a spurious problem. Most of the calculations were performed using the LAOG computer facilities we thank Ginette Buisson for assisting us in the installation of the software.

\section{Appendix}

In Dutrey et al. (1994), we use the following parametrizations for the physical laws in the type 0 model:

Kinetic temperature:

$T_{k}=T_{\mathrm{o}}\left(r / r_{\mathrm{o}}\right)^{-q}$.

Velocity:

$v(r)=v_{\mathrm{o}}\left(r / r_{\mathrm{o}}\right)^{-v}$, Keplerian case: $v=0.5$.

Surface density:

$\Sigma(r)=\Sigma_{\mathrm{o}}\left(r / r_{\mathrm{o}}\right)^{-p}$.

Volume density:

$$
\begin{aligned}
n(r) & =\frac{\Sigma(r)}{\sqrt{\pi} \cdot H(r)} \\
& =n_{\mathrm{o}}\left(r / r_{\mathrm{o}}\right)^{-s} \\
s & =h+p=1+v+p-q / 2 \\
n(r, z) & =n(r) \mathrm{e}^{-\left(\frac{z}{H(r)}\right)^{2}} .
\end{aligned}
$$

Hydrostatic scale height:

$$
\begin{aligned}
H(r) & =\sqrt{\frac{2 k r^{3} T_{k}(r)}{G M_{*} m_{\mathrm{o}}}} \\
& =\sqrt{\frac{2 k}{m_{\mathrm{o}}}} \frac{r}{v(r)} \sqrt{T_{k}(r)} \\
& =H_{\mathrm{o}}\left(r / r_{\mathrm{o}}\right)^{h} \\
h & =1+v-q / 2 .
\end{aligned}
$$

This also implies that $H(r)=\sqrt{2} C_{\mathrm{s}} / \Omega$ (where $C_{\mathrm{s}}$ is the sound speed). $G, M_{*}$ and $m_{\mathrm{o}}$ are the gravitational constant, the stellar mass and the mean molecular weight, respectively.

For the $m_{\mathrm{o}}$, we take the value of $2 \times 1.3 m_{\mathrm{H}}$ where $m_{\mathrm{H}}$ is the hydrogen mass (Beckwith \& Sargent 1993).

Note that other groups (in particular those working on theoretical dust disk modelling) usually use the following definition for $H(r)=C_{\mathrm{s}} / \Omega$, which leads to:

$n(r, z)=n_{\mathrm{o}} \mathrm{e}^{-\left(\frac{z}{2 H(r)}\right)^{2}}$.

This is the case for the definition of $H$ given in Chiang \& Goldreich (1997) and d'Alessio et al. (1998). Therefore, comparing both scale heights imply that "our $H$ " is $\sqrt{2}$ larger. Note that the scale heights presented for comparison in the Fig. 9 take into account this factor. 


\section{References}

D’Alessio, P., Calvet, N., Hartmann, L., Lizano, S., \& Cantó, J. 1999, ApJ, 527, 893 (dA99)

Beckwith, S. V. W., Sargent, A. I., Chini, R. S., \& Guesten, R. 1990, AJ, 99, 924

Beckwith, S. V. W., \& Sargent, A. I. 1993, ApJ, 402, 280

Chiang, E. I., \& Goldreich, P. 1997, ApJ, 490, 368 (CG97)

van Dishoeck, E. F., \& Black, J. H. 1988, ApJ, 334, 771

Draine, B. T., \& Lee, H. M. 1984, ApJ, 285, 89

Dutrey, A., Guilloteau, S., \& Simon, M. 1994, A\&A, 286, 149 (D94)

Dutrey, A., Guilloteau, S., \& Guélin, M. 1997, A\&A, 317, L55 (DGG97)

Elias, J. H. 1978, ApJ, 224, 857
Glassgold, A. E., Huggins, P. J., \& Langer, W. D. 1985, ApJ, 290, 615 Guilloteau, S., \& Dutrey, A. 1994, A\&A, 291, L23

Guilloteau, S., \& Dutrey, A. 1998, A\&A, 339, 467 (GD98)

Koerner, D. W., Sargent, A. I., \& Beckwith, S. V. W. 1993, Icarus, 106, 2 (K93)

Kruegel E., \& Siebenmorgen R. 1994, A\&A, 288, 929

Langer, W. D., \& Penzias, A. A. 1993, ApJ, 408, 539

Lynden-Bell, D., \& Pringle, J. E. 1974, MNRAS, 168, 603

Mannings, V., \& Sargent, A. I. 1997, ApJ, 490, 792

Mathis, J. S., Rumpl, W., \& Nordsieck, K. H. 1977, ApJ, 217, 425

Pietu, V., et al. 2002, A\&A, submitted

Shakura, N. I., \& Sunyaev, R. A. 1973, A\&A, 24, 337

Simon, M., \& Prato, L. 1995, ApJ, 450, 824

Simon, M., Dutrey, A., \& Guilloteau, S. 2000, ApJ, 545, 1034 